\title{
Darcy-scale phase equilibrium modeling with gravity and capillarity
}

\author{
Shuyu Sun ${ }^{\mathrm{a}, *}$ \\ ${ }^{a}$ Physical Science and Engineering Division, King Abdullah University of Science and Technology, Thuwal, \\ 23955-6900, Saudi Arabia
}

\begin{abstract}
The modeling of multiphase fluid mixture and its flow in porous media is of great interest in the field of reservoir simulation. In this paper, we formulate a novel energy-based framework to model multi-component two-phase fluid systems at equilibrium. Peng-Robinson equation of state (EOS) is used to model the bulk properties of each phase, though our framework works well also with other equations of state. Our model reduces to the conventional compositional grading if restricted to one spatial vertical dimension together with the assumption of monodisperse pore-size distribution (all pores being one size). However, our model can be combined with a general distribution of pore size, which can generate interesting behaviors of capillarity in porous media. In particular, the model can be used to predict the capillary pressure of two-phase fluid as a function of saturation, with a given pore-size distribution. This model is the quantitative study of the first time in the literature for the capillarity of a two-phase fluid with partial miscibility. We proposed an unconditionally stable energydecay numerical algorithm based on convex-concave splitting, which has been demonstrated to be both robust and efficient using numerical examples. To verify our model, we simulate the compositional grading of a binary fluid mixture consisting of carbon dioxide and normal decane. To demonstrate powerful features of our model, we provide an interesting example of fluid mixture in a porous medium with wide pore size distribution, where the competition of capillarity and gravity is observed. This work represents the first effort in the literature that rigorously incorporates capillarity and gravity effects into EOS-based phase equilibrium modeling.
\end{abstract}

Keywords: Reservoir simulation, Interfacial tension, Convex splitting, Peng-Robinson equation of state, Diffuse interface models

\section{Introduction}

Two-phase and multi-phase flows are important and common phenomena in petroleum industry, where oil, gas and water are often produced and transported together. In particular, engineers and researchers in reservoir engineering study drainage problems arising during

\footnotetext{
* Corresponding author.

Email address: shuyu.sun@kaust.edu.sa (Shuyu Sun) 
the development and production of oil and gas reservoirs so as to obtain a high economic recovery, by developing, conducting, and interpolating the simulation of subsurface flows of reservoir fluids, including, for example, water, hydrocarbon, $\mathrm{CO}_{2}$, and $\mathrm{H}_{2} \mathrm{~S}$ in porous geological formation. Field-scale (Darcy-scale) simulation has conventionally and routinely used for this purpose [3, 5, 8, 18, 31, 32, 33, 34, 36, 37, 39]. A number of parameters like relative permeability and capillary pressure are taken as given input functions in Darcy-scale simulation [23, 40, 41, 42]. To study these parameters as well as to obtain deep understanding of porous media flow and transport, researchers develop and utilize pore-scale simulation of multiphase flow, which has been shown to be a great research tool to understand the complex hydrodynamic behaviors of the system.

Bulk phase properties like density and solubility are often modeled by an Equation Of State (EOS) [5, 19, 30]. As the most popular EOS to model and compute the fluid equilibrium property of hydrocarbon fluid and other petroleum fluids, the Peng-Robinson EOS [26] is widely used in reservoir engineering and oil industries. With the Peng-Robinson EOS, the Helmholtz free energy $f_{b}(\mathbf{n})$ of a bulk fluid is determined by

$$
\begin{gathered}
f_{b}(\mathbf{n})=f_{b}^{\text {ideal }}(\mathbf{n})+f_{b}^{\text {excess }}(\mathbf{n}), \\
f_{b}^{\text {ideal }}(\mathbf{n})=R T \sum_{i=1}^{M} n_{i}\left(\ln n_{i}-1\right), \\
f_{b}^{\text {excess }}(\mathbf{n})=-n R T \ln (1-b n)+\frac{a(T) n}{2 \sqrt{2} b} \ln \left(\frac{1+(1-\sqrt{2}) b n}{1+(1+\sqrt{2}) b n}\right) .
\end{gathered}
$$

Here $R$ is the universal gas constant (approximately $8.31432 \mathrm{~J} \mathrm{~K}^{-1} \mathrm{~mol}^{-1}$ ) and $T$ is the fluid temperature. In this EOS, we use two parameters, i.e. the energy parameter $a=a(T)$ the covolume parameter $b$, to model the non-ideality of the fluid from the ideal gas law. For completeness, Appendix A contains details on the calculation of the two parameters.

Even though bulk phase properties have been successfully predicted by EOS, interfacial tension [2, 24] is not modeled by a conventional EOS alone. Phase equilibrium with gravity and capillarity is important for petroleum industries, but its modeling remains challenging. Most work on the modeling of phase equilibrium with gravity and capillarity has been carried out at a free space [7, 9, 10, 11, 12, 13, 14, 15, 16, 17, 27, 28, 38. With this free space setting, the domain of consideration is only the pore space, which differs from the domain of consideration at Darcy's scale that includes both pores and grains. In the freespace models, we do not consider solid within the domain, except possibly on the boundary of the domain, where we could impose various boundary conditions to describe the fluidsolid interaction on the boundary. The free-space modeling of fluid mixture can be useful to describe the pore-scale behavior of fluid mixture in geological formation; in particular, it can be utilized to study the phase behavior of fluid mixture within a single pore of a rock. However, the free-space approaches suffer from the small domain it can afford to treat. In 
this paper, we intend to model phase equilibrium with gravity and capillarity at Darcy's scale.

Popular models on the phase equilibrium behaviors of multi-component multi-phase fluid mixture can be categorized into three different types of approaches. The first approach is to use molecular dynamics simulation or Monte Carlo molecular simulation (see, e.g., [6, 43]), where molecular interaction is explicitly modeled using a force field. The second approach is the sharp interface modeling approach (see, e.g., [22]), where the bulk phases are modeled as 3D entities while the interface between two phases is modeled as a $2 \mathrm{D}$ entity. The third approach is sometimes called the diffuse interface modeling method, where both bulk phases and the interface are modeled as 3D entities (see, e.g., [25, 35]). In particular, the thickness direction of the interface is modeled. The diffuse interface modeling method is much cheaper than molecular simulation approaches, but it contains more physics in its framework as compared to the sharp interface models, and consequently it has stronger predition power than the sharp interface models.

Even though this paper is devoted to the modeling of fluid mixture at a Darcy scale, it is helpful to understand certain relevant free-space modeling approaches. Below we briefly review the diffuse interface model for multi-component two-phase fluid mixture based on Peng-Robinson equation of state under possibly influence of gravity and capillarity.

\section{Phase equilibrium modeling with gravity and capillarity at a free space}

\subsection{Mathematical model of the interface between two phases}

We now formulate the calculation of total Helmholtz free energy $F$ involving an interface between two bulk phases. The number of components in the fluid mixture $M$ is given and fixed. The molar concentration of the component $i$ is denoted by $n_{i}$. We let

$$
\mathbf{n}=\left(n_{1}, n_{2}, \cdots, n_{M}\right)^{T}=\frac{\left(N_{1}, N_{2}, \cdots, N_{M}\right)^{T}}{V}
$$

be the molar concentrations of all components and $n=n_{1}+n_{2}+\cdots+n_{N}$ the molar density of the fluid. The total Helmholtz energy density has three contributions, $F_{b}(\mathbf{n})$ from the classical thermodynamic theory of homogeneous bulk fluids, $F_{\nabla}(\mathbf{n})$ from the inhomogeneity of the fluid, and $F_{g}(\mathbf{n})$ from gravity. That is,

$$
\begin{aligned}
F(\mathbf{n}) & =F(\mathbf{n} ; T, \Omega)=\int_{\Omega} f(\mathbf{n} ; T) d \mathbf{x} \\
& =F_{b}(\mathbf{n} ; T, \Omega)+F_{\nabla}(\mathbf{n} ; T, \Omega)+F_{g}(\mathbf{n} ; \Omega) \\
& =\int_{\Omega} f_{b}(\mathbf{n} ; T) d \mathbf{x}+\int_{\Omega} f_{\nabla}(\mathbf{n} ; T) d \mathbf{x}+\int_{\Omega} f_{g}(\mathbf{n}) d \mathbf{x} .
\end{aligned}
$$

Here we denote the contribution of Helmholtz free energy density (i.e. Helmholtz free energy per unit volume of the fluid) from the homogeneous fluid theory by $f_{b}(\mathbf{n})$. Similarly, $F_{\nabla}(\mathbf{n})$ and $F_{g}(\mathbf{n})$ are the contributions of Helmholtz free energy density from the concentration gradient and gravity respectively. We compute the homogeneous term $f_{b}(\mathbf{n})=f_{b}(\mathbf{n} ; T)$ by 
using the EOS approach as described above. With the influence parameter $c_{i j}$, we model the inhomogeneous term (also known as the gradient contribution) $f_{\nabla}(\mathbf{n})$ by a simple but popular quadratic relation:

$$
f_{\nabla}(\mathbf{n})=\frac{1}{2} \sum_{i, j=1}^{M} c_{i j} \nabla n_{i} \cdot \nabla n_{j} .
$$

The parameter $c_{i j}$ is a strong function of temperature but a weak function of molar concentrations. For a given fixed temperature $T$, we might be able to assume $c_{i j}$ to be a constant. The detailed computation for $c_{i j}$ will be described in a later subsection.

We model the gravity contribution $f_{g}(\mathbf{n})$ by

$$
f_{g}(\mathbf{n})=n M_{w} g z
$$

where $z$ is the depth and $g$ is the gravitational acceleration constant (approximately 9.087 $\mathrm{m} / \mathrm{s}^{2}$ ). The molecular weight of the mixture $M_{w}$ is related to the molecular weights of pure substances $M_{w, i}$ and mole fractions $y_{i}$ :

$$
M_{w}=\sum_{i=1}^{M} M_{w, i} y_{i}=\sum_{i=1}^{M} M_{w, i} \frac{N_{i}}{N}=\sum_{i=1}^{M} M_{w, i} \frac{n_{i}}{n} .
$$

Of course, the scenario without gravity can be modeled by simply letting $g=0$ here.

Let $\mathcal{H}$ be a space of functions with certain regularity. According to the second law of thermodynamics, the total Helmholtz free energy achieves its (global) minimum at the equilibrium state in an NVT system, including the cases where we have interfaces between two bulk phases. The mathematical statement of equilibrium can be formulated as follows: To find $\mathbf{n} \in \mathcal{H}$ such that the total Helmholtz free energy $F$ is minimized, subject to the mass conservation constraint. That is,

$$
\min _{\mathbf{n} \in \mathcal{H}} F(\mathbf{n})
$$

subject to

$$
\int_{\Omega} \mathbf{n} d \mathbf{x}=\mathbf{N}^{t}
$$

\subsection{The calculation of the influence parameters}

We model the phase interface by the influence parameters $c_{i j}$. It can be computed from the parameter of pure substance using a certain mixing rule:

$$
c_{i j}=\left(1-\beta_{i j}\right) \sqrt{c_{i} c_{j}} .
$$

Here the parameter $\beta_{i j}$ is the binary interaction coefficient for the influence parameter. We usually assume that $\beta_{i j}$ sits between 0 and 1 and $\beta_{i j}=\beta_{j i}$. In cases of lacking data or for convenience, $\beta_{i j}$ is assumed to be zero. We note that if $\beta_{i j}=0$, the above mixing rule is 
simplified into the simple geometric mean. Certain algorithms can take advantage of the simple geometric mean [9].

We may get the influence parameter of pure substance $c_{i}$ from the Peng-Robinson parameters $a_{i}$ and $b_{i}$, and the accentric factor $\omega_{i}$ by [1, 20, 21]:

$$
c_{i}=a_{i} b_{i}^{2 / 3}\left(m_{1, i}^{c}\left(1-\frac{T}{T_{c_{i}}}\right)+m_{2, i}^{c}\right)
$$

and

$$
\begin{aligned}
m_{1, i}^{c} & =-\frac{10^{-16}}{1.2326+1.3757 \omega_{i}}, \\
m_{2, i}^{c} & =\frac{10^{-16}}{0.9051+1.5410 \omega_{i}} .
\end{aligned}
$$

\subsection{Mathematical formulation using chemical potential}

From thermodynamics [5] and from variational principles [29], we know that the total chemical potential $\mu$ must be a spatially constant:

$$
\mu_{i}^{\text {tot }}(\mathbf{x})=\text { constant }, \forall \mathbf{x} \in \Omega, i=1,2, \cdots, M .
$$

The homogeneous fluid term, the gradient theory term, and the gravity term, all contribute to the total chemical potential:

$$
\mu_{i}^{\text {tot }}=\mu_{b, i}+\mu_{\nabla, i}+\mu_{g, i} .
$$

Since we will use the homogeneous fluid part of chemical potential (the conventional concept of chemical potential) $\mu_{b, i}$ many times throughout the paper, for convenience of notation, we denote it also by $\mu_{i}=\mu_{b, i}$.

We may compute the homogeneous fluid contribution and the gravity contribution by:

$$
\begin{aligned}
\mu_{i} & =\left(\frac{\partial F_{b}}{\partial N_{i}}\right)_{T, V, N_{1}, \cdots, N_{c-1}, N_{c+1}, \cdots, N_{M}}=\left(\frac{\partial f_{b}}{\partial n_{i}}\right)_{T, n_{1}, \cdots, n_{c-1}, n_{c+1}, \cdots, n_{M}}, \\
\mu_{g, i} & =\left(\frac{\partial f_{g}}{\partial n_{i}}\right)_{T, n_{1}, \cdots, n_{c-1}, n_{c+1}, \cdots, n_{M}}=M_{w, i} g z .
\end{aligned}
$$

With variational calculus, one derives the gradient contribution of chemical potential as:

$$
\mu_{\nabla, i}=\frac{1}{2} \sum_{k, j=1}^{N} \frac{\partial c_{k j}}{\partial n_{i}} \nabla n_{k} \cdot \nabla n_{j}-\sum_{j=1}^{M} \nabla \cdot\left(c_{i j} \nabla n_{j}\right) .
$$

Frequently, people assume that the influence parameter $c_{i j}$ is independent of the molar concentration $\mathbf{n}$. With this assumption, the chemical equilibrium condition (7) implies

$$
-\sum_{j=1}^{M} c_{i j} \nabla^{2} n_{j}=\mu_{i}^{\mathrm{tot}}-\mu_{i}(\mathbf{n})-M_{w, i} g z, \quad i=1,2, \cdots, M .
$$

Here the total chemical potential of component $\mu_{i}^{\text {tot }}$ is a unknown scalar constant, which can be solved together with the unknown molar density variable $\mathbf{n}$ using (8) and the equation of global mass conservation law. 


\section{A new framework for Darcy-scale modeling of phase equilibrium behaviors}

\subsection{Description of porous media: pore-size distribution}

It is well known that the pore space, including pore geometry and pore structure, affects greatly both the fluid flow in porous media and the equilibrium behaviors of the fluid within porous media. In this paper, we consider two important quantities of porous media in our model. The first one is the distribution of pore size, and the second one is the interfacial tension between the solid wall and the fluid phase within the pore space. It is well known that the pore-size distribution of a porous medium influences porosity and permeability, and in this paper, we will see how it influences quantitatively the phase behaviors of fluid mixture within the porous medium.

Grain size distribution has been used to describe porous media in the literature. Our interested quantity from the perspective of modeling fluid mixture within porous media (as well as the flow through porous media), however, is not the direct properties of the matrix itself (e.g. grain size distribution) but rather the properties of the pores (e.g. pore size distribution). It is easy to imagine that the cross sectional dimensions of the pore space in a rock will change greatly in shape and in size as one moves through the pore space. People sometimes distinguish pore space by the size of the cross sectional areas, and narrow passages are called pore throats, which usually separated by wide passages called pore bodies.

Since the cross sectional area of the pore space is not necessarily in a circular shape, we define effective pore size using specific surface area. Specific surface area is a property of porous media defined as the total (inner) surface area of a rock per unit of pore volume. Consider a circular tube of radius $r$ and length $L$, the (inner) surface area is $S=2 \pi r L$ while the pore volume is $V=\pi r^{2} L$. The specific surface area is

$$
A_{s}(r)=\frac{S}{V}=\frac{2 \pi r L}{\pi r^{2} L}=\frac{2}{r} .
$$

For pore space with non-circular cross sectional areas, we use the following equation to define $r_{\text {eff }}$ via the specific surface area. We denote the effective radius also by $r$ in the rest of the paper for convenience.

$$
r=r_{\mathrm{eff}}=\frac{2}{A_{s}}
$$

For example, for a rectangular prism of sides $a$ and $b$ and height $h$, the specific area is

$$
A_{s}=\frac{S}{V}=\frac{2(a+b) h}{a b h}=\frac{2(a+b)}{a b} .
$$

Then its effective radius is

$$
r_{\mathrm{eff}}=\frac{2}{A_{s}}=\frac{a b}{a+b}
$$

Pore size distribution of porous media can be measured using using various types of microscopy on thin sections or other flat soil surfaces, or tomographs, and then followed by computerized geometric analysis of digitized images of individual pores. Other common 
methods of measuring pore-size distribution include those based on effective capillary size, for example, the water-retention based method and the method of mercury porosimetry. These techniques can yield fluid content $\theta$ of the porous medium as a function of the imposed pressure $p$, known as the fluid retention curve. By using the Young-Laplace equation, one can convert the imposed pressure $p$ to the pore size $r$. With this conversion, the fluid retention curve yields a cumulative pore-size distribution function, which we denote by $\theta(r)$ here. The pore-size density function $\psi(r)$ is simply the derivative of cumulative pore-size distribution function with respect to the pore size.

$$
\psi(r):=\frac{d \theta(r)}{d r} .
$$

We note that $\theta(r)$ is a increasing function of $r$. Obviously the maximum value of $\theta(r)$ is porosity. Let us denote the range of pore size by $\Gamma \subset[0,+\infty)$. We then have

$$
\max _{r \in \Gamma} \theta(r)=\phi
$$

which implies

$$
\int_{r \in \Gamma} \psi(r)=\phi
$$

The above concept of pore-size density function $\psi(r)$ is for a homogeneous porous medium. For a heterogeneous porous medium, the pore-size distribution can vary spatially. That is, both the cumulative pore-size distribution function and the pore-size density function can be a function of the spatial position $\mathbf{x} \in \Omega$ and pore size $r \in \Gamma$, where $\Omega \subset \mathbb{R}^{3}$ is the domain occupied by the porous medium. The relation between the two variables $\psi$ and $\theta$ still remains, but with a partial derivative:

$$
\psi(\mathbf{x}, r):=\frac{\partial \theta(\mathbf{x}, r)}{\partial r}
$$

Integrating the pore-size density function over all possible pore size, we still should get the porosity, now as a function of position:

$$
\int_{r \in \Gamma} \psi(\mathbf{x}, r)=\phi(\mathbf{x})
$$

\subsection{Description of the interaction between porous media and fluids: interfacial tension}

In addition to the pore space itself, the fluid-solid interaction has also a great influence on both the fluid flow in porous media and the equilibrium behaviors of the fluid within the porous media. Parameters in fluid-solid interaction include wettability, contact angle and interfacial tension between the fluid and solid phases.

Wettability is the tendency of one fluid to spread on, or degree of one fluid to adhere to, a solid surface in the presence of another immiscible fluid or other immiscible fluids, and it represents the interaction between fluid and solid phases. In a subsurface reservoir rock, the solid phase is the rock mineral assemblage while the fluid phase can be water or oil or 
gas. The contact angle of a solid-liquid-vapor system is the angle between the liquidvapor interface and the liquid-solid interface at the contact line of the three phases where both liquid and gas phases meets the solid surface. This equilibrium contact angle quantifies the wettability of the solid surface by the liquid phase relatively to the gas phase. At a given temperature and pressure, a system of a given solid, together with liquid and vapor with given composition has a unique equilibrium contact angle.

The concepts of wettability, contact angle and interfacial tension are highly related. In fact, by modeling interfacial tension properly, we also model wettability and contact angle. We denote by $\sigma_{s v}$ the solid-vapor interfacial tension (i.e. the solid-vapor interfacial energy per contact area), by $\sigma_{s l}$ the solidliquid interfacial tension (i.e. the solidliquid interfacial energy per contact area), and by $\sigma_{l v}$ the liquid-vapor interfacial tension. By using the minimal Helmholtz principle or by using a simple force balance on the direction along the solid-fluid plane, one can derive the following Young's equation which relates the equilibrium contact angle $\theta_{\text {contact }}$ with these three interfacial tensions:

$$
\sigma_{s v}-\sigma_{s l}-\sigma_{l g} \cos \theta_{\text {contact }}=0
$$

As seen from the energy formulation of fluid mixture discussed in the previous section, our fluid mixture theory allows fluid molar density to vary continuously between the two bulk phases of liquid and vapor. Moreover, our fluid mixture model does not explicitly mark whether a phase is fluid or vapor; instead, we track only molar concentration and molar density and we use molar concentration/density as our order parameters to distinguish phases. Therefore, instead of providing the solid-liquid interfacial tension $\sigma_{s l}$ and the solidvapor interfacial tension $\sigma_{s v}$, we need to provide a more general solid-fluid interfacial tension as a function of molar concentration of the fluid, which we denote as $\sigma_{s f}=\sigma_{s f}(\mathbf{n})$. Of course, if the molar concentration picks the value of the bulk liquid phase, the solid-fluid interfacial tension $\sigma_{s l}$ should have the same value as the solid-liquid interfacial tension $\sigma_{s l}$; that is,

$$
\sigma_{s f}\left(\mathbf{n}^{L}\right)=\sigma_{s l}
$$

Similarly, the solid-fluid interfacial tension $\sigma_{s l}$ equals to the solid-vapor interfacial tension $\sigma_{s l}$ when the fluid is the vapor:

$$
\sigma_{s f}\left(\mathbf{n}^{V}\right)=\sigma_{s v} .
$$

The values of $\sigma_{s l}$ and $\sigma_{s v}$ are widely available in the literature for various fluid-solid systems. However, the solid-fluid interfacial tension $\sigma_{s l}$ as a function of $\mathbf{n}$ has not been well reported. The solid-fluid interfacial tension $\sigma_{s l}$ can be obtained by either performing molecular simulation or by fitting macro-scale experimental data. Our preliminary numerical investigation indicated that the Darcy-scale phase behavior of the fluid mixture in porous media is not very sensitive to the specific function form of the solid-fluid interfacial tension $\sigma_{s l}$ as long as the values of $\sigma_{s f}\left(\mathbf{n}^{L}\right)$ and $\sigma_{s f}\left(\mathbf{n}^{V}\right)$ are properly imposed.

In this paper, we propose three simple models for the solid-fluid interfacial tension $\sigma_{s f}$. 
The first model assumes a form of piece-wise constant function:

$$
\sigma_{s f}(\mathbf{n})= \begin{cases}\sigma_{s l}, & \text { if } \sum_{i=1}^{M} n_{i} \leq n^{L}, \\ \sigma_{s v}, & \text { if } \sum_{i=1}^{M} n_{i}>n^{L} .\end{cases}
$$

The second model is a linear function of overall molar density $n:=\sum_{i=1}^{M} n_{i}$ :

$$
\sigma_{s f}(\mathbf{n})=\frac{\left(\sum_{i=1}^{M} n_{i}-n^{V}\right) \sigma_{s l}+\left(n^{L}-\sum_{i=1}^{M} n_{i}\right) \sigma_{s v}}{n^{L}-n^{V}} .
$$

The third model is given by the hyperbolic tangent function:

$$
\sigma_{s f}(\mathbf{n})=\left(\frac{\sigma_{s l}+\sigma_{s v}}{2}\right)+\left(\frac{\sigma_{s l}-\sigma_{s v}}{2}\right) \tanh \left(\frac{\alpha\left(\sum_{i=1}^{M} n_{i}-\frac{n^{L}+n^{V}}{2}\right)}{n^{L}-n^{V}}\right),
$$

where the model parameter $\alpha>0$ controls the shape of the function. The bigger the parameter $\alpha$ is, the closer this model is to the piece-wise constant function model. We suggest to choose $\alpha=3$.

\subsection{Energy-based framework of fluid mixture in porous media}

We model the phase behavior of multi-component (possibly two-phase) fluid mixture in porous media by specifying the NVT (i.e. the moles of each species, the volume of the fluid mixture, and the temperature of the system). According to the theory of equilibrium thermodynamics, the total Helmholtz free energy $F^{\text {tot }}$ of the system achieves its minimum at equilibrium. To model the equilibrium phase behavoir of the system, we will then need to specify how the total Helmoholtz free energy of the system varies with molar concentration $n_{i}$ of each species $i$, the volume of the system, and the temperature of the system. By considering only the isothermal scenarios in a fixed domain (thus temperature and volume to remain constants), we focus mainly on how the total Helmoholtz free energy $F^{\text {tot }}$ changes with molar concentration $n_{i}$ of each species $i$. Since molar concentration $n_{i}$ is a function of spatial position itself, the dependence of the total Helmoholtz free energy $F^{\text {tot }}$ on molar concentration $n_{i}$ is a functional (not a function), a mapping from a space of functions into the real numbers. The total Helmoholtz free energy $F^{\text {tot }}$ has contributions from bulk fluid, liquid-vapor interface, fluid-solid interface, and gravity:

$$
F^{\mathrm{tot}}[\mathbf{n}]=F_{b}[\mathbf{n}]+F_{l v}[\mathbf{n}]+F_{s f}[\mathbf{n}]+F_{g}[\mathbf{n}],
$$

where for clarity, square brackets are used for functionals while parenthesis for classical functions. 
The bulk contribution $F_{b}[\mathbf{n}]$ can be calculated by

$$
F_{b}[\mathbf{n}]=\int_{V} \int_{\Gamma} f_{b}(\mathbf{n}(\mathbf{x}, r)) \psi(\mathbf{x}, r) d r d \mathbf{x}
$$

where the bulk Helmholtz free energy density function $f_{b}(\mathbf{n})$ can be modeled using PengRobinson equation of state (or any other equation of state) as we discussed in the previous section.

At a pore scale, $F_{l v}=F_{\nabla}$ is modeled using the gradient theory. At the Darcy scale, we model it using a sharp-interface approach:

$$
F_{l v}[\mathbf{n}]=\int_{A_{l v}} \sigma_{l v} d A
$$

where $A_{l v}$ is the liquid-vapor interface, and $\sigma_{l v}$ is the liquid-vapor interfacial tension, i.e. the Helmoholtz free energy of the interface per area. Rigorously, $\sigma_{l v}$ can be a function of pressure and composition of the two adjacent phases. In the cases that we are able to assume it to be a constant, we may write

$$
F_{l v}[\mathbf{n}]=A_{l v} \sigma_{l v},
$$

where $A_{l v}$ now means the total area of the liquid-vapor interface.

Modeling of the fluid-solid interface contribution $F_{s f}[\mathbf{n}]$ is an important piece of our framework, because $F_{s f}[\mathbf{n}]$ can be much larger than $F_{l v}[\mathbf{n}]$, and the fluid-solid interface contribution can greatly affect our predicted phase behaviors. Like the sharp-interface approach we used in liquid-vapor interface, the fluid-solid interface contribution $F_{s f}[\mathbf{n}]$ is modeled by

$$
F_{s f}[\mathbf{n}]=\int_{A_{s f}} \sigma_{s f}(\mathbf{n}) d A
$$

where the function $\sigma_{s f}(\mathbf{n})$ satisfies $\sigma_{s f}\left(\mathbf{n}^{L}\right)=\sigma_{s l}$ and $\sigma_{s f}\left(\mathbf{n}^{V}\right)=\sigma_{s v}$.

For certain simplified cases, we may assume both $\sigma_{s l}$ and $\sigma_{s v}$ to be constant. In these cases, we may split the fluid-solid interface $A_{s f}$ into two pieces, $A_{s l}$ and $A_{s v}$; that is, $A_{s f}=A_{s l} \bigcup A_{s v}$. Under this assumption, we may write the surface (the fluid-solid interface) contribution as

$$
F_{s f}[\mathbf{n}]=\int_{A_{s l}} \sigma_{s l} d s+\int_{A_{s v}} \sigma_{s v} d s=A_{s l} \sigma_{s l}+A_{s v} \sigma_{s v}
$$

Modeling of the gravity contribution is straightforward:

$$
F_{g}[\mathbf{n}]=\int_{V} \int_{\Gamma}(-\mathbf{g} \cdot \mathbf{x}) \sum_{i=1}^{M} M_{w, i} n_{i} \psi(\mathbf{x}, r) d r d \mathbf{x},
$$

where $\mathbf{g}$ the gravitational acceleration near Earth's surface, and $M_{w, i}$ is the molecular weight of species $i$. 
In the above equation (11), the term involves an area integration $\left(\int_{A_{s f}} \sigma_{s f}(\mathbf{n})\right)$, which is not convenient to manipulate. We thus convert it to a volume integration by using the specific surface area $A_{s}$ and our pore-size density function $\psi$ defined earlier:

$$
F_{s f}=\int_{A_{s f}} \sigma_{s f}(\mathbf{n})=\int_{V} \int_{\Gamma} A_{s}(r) \sigma_{s f}(\mathbf{n}(\mathbf{x}, r)) \psi(\mathbf{x}, r) d r d \mathbf{x} .
$$

With this, equation 10 becomes

$$
\begin{aligned}
& F^{\mathrm{tot}}[\mathbf{n}] \\
= & F_{b}[\mathbf{n}]+A_{l v} \sigma_{l v}+\int_{V} \int_{\Gamma} A_{s}(r) \sigma_{s f}(\mathbf{n}) \psi(\mathbf{x}, r) d r d \mathbf{x}+\int_{V} \int_{\Gamma}(-\mathbf{g} \cdot \mathbf{x}) \sum_{i=1}^{M} M_{w, i} n_{i} \psi(\mathbf{x}, r) d r d \mathbf{x} \\
= & A_{l v} \sigma_{l v}+\int_{V} \int_{\Gamma}\left(f_{b}(\mathbf{n})+A_{s}(r) \sigma_{s f}(\mathbf{n})-(\mathbf{g} \cdot \mathbf{x}) \sum_{i=1}^{M} M_{w, i} n_{i}\right) \psi(\mathbf{x}, r) d r d \mathbf{x},
\end{aligned}
$$

where we have suppressed the dependence of $\mathbf{n}$ on $\mathbf{x}$ and $r$ for clarity.

Again, let $\mathcal{H}$ be a space of functions with certain regularity. The mathematical statement of equilibrium for this multi-component two-phase fluid mixture in a porous medium can be formulated as follows. We seek $\mathbf{n} \in \mathcal{H}$ to minimize the total Helmholtz free energy $F$ :

$$
\min _{\mathbf{n} \in \mathcal{H}} F^{\mathrm{tot}}(\mathbf{n})=\min _{\mathbf{n} \in \mathcal{H}}\left(F_{b}[\mathbf{n}]+F_{l v}[\mathbf{n}]+F_{s f}[\mathbf{n}]+F_{g}[\mathbf{n}]\right),
$$

subject to the mass conservation constraint

$$
\int_{V} \int_{\Gamma} \mathbf{n}(\mathbf{x}, r) \psi(\mathbf{x}, r) d r d \mathbf{x}=\mathbf{N}^{\text {tot }}
$$

\subsection{Stationarity condition for phase equilibrium}

We apply variational calculus and the method of Lagrange's undetermined multipliers for finding the minimum of the total Helmholtz free energy (12) subject to the equality constraint (13). We introduce a new variable $\boldsymbol{\mu}^{\text {tot }}=\left\{\mu_{i}^{\text {tot }}, i=1,2, \cdots, M\right\}$ known as the Lagrange undetermined multiplier and we formulate the following Lagrange function defined by

$$
\begin{aligned}
& L_{F}\left[\mathbf{n}, \boldsymbol{\mu}^{\mathrm{tot}}\right]:=F[\mathbf{n}]-\sum_{i=1}^{M} \mu_{i}^{\mathrm{tot}}\left(\int_{V} \int_{\Gamma} n_{i}(\mathbf{x}, r) \psi(\mathbf{x}, r) d r d \mathbf{x}-N_{i}^{\mathrm{tot}}\right) \\
= & \int_{V} \int_{\Gamma}\left(f_{b}(\mathbf{n})+A_{s}(r) \sigma_{s f}(\mathbf{n})-(\mathbf{g} \cdot \mathbf{x}) \sum_{i=1}^{M} M_{w, i} n_{i}-\sum_{i=1}^{M} \mu_{i}^{\mathrm{tot}} n_{i}\right) \psi(\mathbf{x}, r) d r d \mathbf{x} \\
& +A_{l v} \sigma_{l v}+\sum_{i=1}^{M} \mu_{i}^{\mathrm{tot}} N_{i}^{\mathrm{tot}} .
\end{aligned}
$$


We assume that $A_{l v} \sigma_{l v}$ is a constant. Then the variational derivative of $L_{F}\left[\mathbf{n}, \boldsymbol{\mu}^{\text {tot }}\right]$ with respect to $\mathbf{n}$ is

$$
\begin{aligned}
& \frac{\delta L_{F}\left[\mathbf{n}, \boldsymbol{\mu}^{\mathrm{tot}}\right]}{\delta n_{i}} \\
= & \frac{\partial}{\partial n_{i}}\left(\left(f_{b}(\mathbf{n})+A_{s}(r) \sigma_{s f}(\mathbf{n})-(\mathbf{g} \cdot \mathbf{x}) \sum_{i=1}^{M} M_{w, i} n_{i}-\sum_{i=1}^{M} \mu_{i}^{\mathrm{tot}} n_{i}\right) \psi(\mathbf{x}, r)\right) \\
= & \left(\mu_{i}(\mathbf{n})+A_{s}(r) \mu_{i}^{\sigma}(\mathbf{n})-(\mathbf{g} \cdot \mathbf{x}) M_{w, i}-\mu_{i}^{\mathrm{tot}}\right) \psi(\mathbf{x}, r),
\end{aligned}
$$

where we have used the following well known relationship between chemical potential and Helmholtz free energy density: $\mu_{i}=\frac{\partial f_{b}}{\partial n_{i}}$. Here we have also defined

$$
\mu_{i}^{\sigma}:=\frac{\partial \sigma_{s f}(\mathbf{n})}{\partial n_{i}} .
$$

The stationarity condition implies

$$
\mu_{i}(\mathbf{n}(\mathbf{x}, r))+A_{s}(r) \mu_{i}^{\sigma}(\mathbf{n}(\mathbf{x}, r))-(\mathbf{g} \cdot \mathbf{x}) M_{w, i}=\mu_{i}^{\text {tot }},
$$

for all meaningful pair $(\mathbf{x}, r)$ and all species $i$. Meaningful pair $(\mathbf{x}, r)$ refers to the pair such that $\psi(\mathbf{x}, r)>0$. We see that the Helmholtz free energy of the liquid-vapor interface does not enter the final stationarity condition (14). This implies that we can ignore the Helmholtz free energy of the liquid-vapor interface in our model. We will discuss about its implication further in later sections.

\section{Discussion and analysis of a few simplified scenarios}

\subsection{Homogeneous porous media but with a certain pore-size distribution}

We first consider an important special case, a homogeneous porous medium. That is, in a volume $V_{0}$ of the porous medium, all parameters of the medium is constant spatially. We also ignore the gravity effect. However, we allow the pores within the medium to follow a certain given (possibly non-monodisperse) pore-size distribution; in other words, the pore size does not have to be a single value, as we allow both large pores and small pores. This special case can also be applied to a single spatial location of the domain at the Darcy's scale, if the porous media is heterogeneous; in this case, the volume $V_{0}$ can be viewed as a infinitesimal volume containing the single spatial location of our focus.

Within $V_{0}$, the pore-size density function is now spatially independent:

$$
\psi(\mathbf{x}, r)=\psi(r)
$$


Ignoring the contribution of the liquid-vapor interface $F_{l v}[\mathbf{n}]$ and the gravity effect $F_{g}[\mathbf{n}]$, we have

$$
\begin{aligned}
F^{\mathrm{tot}}[\mathbf{n}] & =F_{b}[\mathbf{n}]+F_{s f}[\mathbf{n}] \\
& =\int_{V_{0}} \int_{\Gamma} \psi(r) f_{b}(\mathbf{n}(r)) d r d \mathbf{x}+\int_{V_{0}} \int_{\Gamma} A_{s}(r) \sigma_{s f}(\mathbf{n}(r)) \psi(r) d r d \mathbf{x} \\
& =\left|V_{0}\right| \int_{\Gamma}\left(f_{b}(\mathbf{n}(r))+A_{s}(r) \sigma_{s f}(\mathbf{n}(r))\right) \psi(r) d r
\end{aligned}
$$

The phase equilibrium problem is to minimize the total Helmholtz free energy $F[\mathbf{n}]$ and it is equivalent to minimize the total Helmholtz free energy density

$$
f^{\mathrm{tot}}[\mathbf{n}]:=\frac{F^{\mathrm{tot}}[\mathbf{n}]}{\left|V_{0}\right|}=\int_{\Gamma}\left(f_{b}(\mathbf{n}(r))+A_{s}(r) \sigma_{s f}(\mathbf{n}(r))\right) \psi(r) d r
$$

subject to the following mass conservation constraints:

$$
\int_{\Gamma} n_{i}(r) \psi(r) d r=\frac{N_{i}^{\mathrm{tot}}}{V_{0}}, \quad i=1,2, \cdots, M
$$

We again introduce Lagrange's undetermined multiplier $\boldsymbol{\mu}^{\text {tot }}=\left\{\mu_{i}^{\text {tot }}, i=1,2, \cdots, M\right\}$ and formulate the following Lagrangian function

$$
\begin{aligned}
& L_{f}\left[\mathbf{n}, \boldsymbol{\mu}^{\mathrm{tot}}\right]:=f[\mathbf{n}]-\sum_{i=1}^{M} \mu_{i}^{\mathrm{tot}}\left(\int_{\Gamma} n_{i}(r) \psi(r) d r-\frac{N_{i}^{\mathrm{tot}}}{V_{0}}\right) \\
= & \int_{\Gamma}\left(f_{b}(\mathbf{n}(r))+A_{s}(r) \sigma_{s f}(\mathbf{n}(r))\right) \psi(r) d r-\sum_{i=1}^{M} \mu_{i}^{\mathrm{tot}} \int_{\Gamma} n_{i}(r) \psi(r) d r+\sum_{i=1}^{M} \frac{\mu_{i}^{\mathrm{tot}} N_{i}^{\mathrm{tot}}}{V_{0}} .
\end{aligned}
$$

We now consider the variational derivatives of $L_{f}\left[\mathbf{n}, \boldsymbol{\mu}^{\text {tot }}\right]$

$$
\begin{aligned}
& \frac{\delta L_{f}\left[\mathbf{n}, \boldsymbol{\mu}^{\mathrm{tot}}\right]}{\delta n_{i}} \\
= & \frac{\partial}{\partial n_{i}}\left(f_{b}(\mathbf{n}(r)) \psi(r)+A_{s}(r) \sigma_{s f}(\mathbf{n}(r)) \psi(r)-\sum_{k=1}^{M} \mu_{k}^{\mathrm{tot}} n_{k}(r) \psi(r)\right) \\
= & \mu_{i}(\mathbf{n}(r)) \psi(r)+A_{s}(r) \mu_{i}^{\sigma}(\mathbf{n}(r)) \psi(r)-\mu_{i}^{\mathrm{tot}} \psi(r) .
\end{aligned}
$$

Setting the above variational derivative to be zero, we obtain the following stationarity condition:

$$
\mu_{i}(\mathbf{n}(r))+A_{s}(r) \mu_{i}^{\sigma}(\mathbf{n}(r))=\mu_{i}^{\text {tot }} .
$$

We recall (9) and conclude

$$
\mu_{i}(\mathbf{n}(r))+\frac{2 \mu_{i}^{\sigma}(\mathbf{n}(r))}{r}=\mu_{i}^{\text {tot }}
$$


Solving the nonlinear algebraic equation 15 for molar concentration $\mathbf{n}$ could give the distribution of the molar concentration among pores of different sizes. After we obtain the distribution of molar concentration among pores of different sizes, we then quickly recover the chemical potential of each species among pores of different sizes simply using our closedform formula of chemical potential derived from Peng-Robinson equation of state. Then we calculate the distribution of pressure among pores of different sizes using the fact (see Appendix B for its derivation) that

$$
p=\sum_{i=1}^{M} \mu_{i}(\mathbf{n}) n_{i}-f_{b}(\mathbf{n}) .
$$

The result of molar density and pressure can further yield to the saturation value of the system and the pressure jump between the liquid and vapor phases. By varying the given $\mathbf{N}^{\text {tot }}$, we can then predict that curve of capillary pressure as a function of saturation, one of the key inputs for Darcy-scale multiphase flow simulation in porous media.

\subsection{A further simplification of homogeneous porous media}

We use molar concentrations and density as the order parameters for our phase identification. If pressure and temperature are constant, molar density and molar concentrations remain constant in bulk phases at equilibrium, and they varies only within and near the liquid-vapor interface. We consider only isothermal systems in this paper; hence temperature is a given constant. We further assume that pressure is constant in each of the liquid and vapor phases. If we view the system from a sharp-interface point of view, we may see that the molar concentration $\mathbf{n}$ picks only two values: it equals to the constant $\mathbf{n}^{L}$ in the liquid phase and it takes the constant $\mathbf{n}^{V}$ in the vapor phase. We now define the liquid region and the vapor region by

$$
\begin{aligned}
& \Gamma_{L}:=\left\{r \in \Gamma: \sum_{i=1}^{M} n_{i}(r) \geq \frac{n^{L}+n^{V}}{2}\right\}, \\
& \Gamma_{V}:=\left\{r \in \Gamma: \sum_{i=1}^{M} n_{i}(r)<\frac{n^{L}+n^{V}}{2}\right\} .
\end{aligned}
$$

With our assumption, the function $\mathbf{n}$ becomes the following piece-wise constant function:

$$
\mathbf{n}= \begin{cases}\mathbf{n}^{L}, & \text { if } r \in \Gamma_{L}, \\ \mathbf{n}^{V}, & \text { if } r \in \Gamma_{V} .\end{cases}
$$

This indicates that the entire function $\mathbf{n}$ is determined by three parameters $\mathbf{n}^{L}, \mathbf{n}^{V}$, and $\Gamma_{L}$, because $\Gamma_{L}=\Gamma \backslash \Gamma_{V}$.

It is easy to see the pore space occupied by the liquid phase will be

$$
V_{\text {liquid }}=V_{0} \int_{r \in \Gamma_{L}} \psi(r) d r
$$


The integral on the right-hand side of this equation means the liquid phase content, which divided by porosity yields to the liquid phase saturation:

$$
S_{L}=\frac{1}{\phi} \int_{r \in \Gamma_{L}} \psi(r) d r
$$

Similarly, we have the vapor phase saturation:

$$
S_{V}=\frac{1}{\phi} \int_{r \in \Gamma_{V}} \psi(r) d r
$$

Obviously, we have the summation relationship: $S_{L}+S_{V}=1$, because $\phi=\int_{r \in \Gamma} \psi(r) d r$.

The total Helmholtz free energy density $f^{\text {tot }}[\mathbf{n}]:=\frac{F^{\text {tot }}[\mathbf{n}]}{\left|V_{0}\right|}$ is a functional of molar concentration $\mathbf{n}$. Since $\mathbf{n}$ is defined by the three parameters $\mathbf{n}^{L}, \mathbf{n}^{V}$, and $\Gamma_{L}$, the total Helmholtz free energy density is also fully determined by three parameters $\mathbf{n}^{L}, \mathbf{n}^{V}$, and $\Gamma_{L}$. By assuming the domain $\Gamma^{L}$ can be parameterized using one or a few real numbers (for example $\Gamma^{L}=\left[r_{0}^{L}, r_{1}^{L}\right]$ ), the total Helmholtz free energy density is now a function (not a functional anymore) of $\mathbf{n}^{L}, \mathbf{n}^{V}$ and $\Gamma^{L}$ :

$$
f^{\mathrm{tot}}\left(\mathbf{n}^{L}, \mathbf{n}^{V}, \Gamma^{L}\right)=\int_{\Gamma}\left(f_{b}(\mathbf{n}(r))+A_{s}(r) \sigma_{s f}(\mathbf{n}(r))\right) \psi(r) d r .
$$

Splitting $\Gamma$ into $\Gamma_{L}$ and $\Gamma_{V}$, we obtain

$$
\begin{aligned}
& f^{\mathrm{tot}}\left(\mathbf{n}^{L}, \mathbf{n}^{V}, \Gamma_{L}\right) \\
= & \int_{\Gamma_{L}} f_{b}(\mathbf{n}) \psi(r) d r+\int_{\Gamma_{V}} f_{b}(\mathbf{n}) \psi(r) d r+\int_{\Gamma_{L}} A_{s}(r) \sigma_{s f}(\mathbf{n}) \psi(r) d r+\int_{\Gamma_{V}} A_{s}(r) \sigma_{s f}(\mathbf{n}) \psi(r) d r . \\
= & \phi S_{L} f_{b}\left(\mathbf{n}^{L}\right)+\phi S_{V} f_{b}\left(\mathbf{n}^{V}\right)+\sigma_{s l} \int_{\Gamma_{L}} A_{s}(r) \psi(r) d r+\sigma_{s v} \int_{\Gamma_{V}} A_{s}(r) \psi(r) d r .
\end{aligned}
$$

The formulation is to miminze $f^{\text {tot }}\left(\mathbf{n}^{L}, \mathbf{n}^{V}, \Gamma_{L}\right)$ subject to the following mass conservation constraints:

$$
\int_{\Gamma_{L}} n_{i}(r) \psi(r) d r+\int_{\Gamma_{V}} n_{i}(r) \psi(r) d r=\frac{N_{i}^{\mathrm{tot}}}{V_{0}}, \quad i=1, \cdots, M
$$

which are

$$
\phi\left(S_{L} n_{i}^{L}+S_{V} n_{i}^{V}\right)=\frac{N_{i}^{\text {tot }}}{V_{0}}, \quad i=1, \cdots, M
$$


The corresponding Lagrangian function becomes

$$
\begin{aligned}
& L_{f}\left(\mathbf{n}^{L}, \mathbf{n}^{V}, \Gamma_{L}, \boldsymbol{\mu}^{\mathrm{tot}}\right) \\
:= & f^{\mathrm{tot}}\left(\mathbf{n}^{L}, \mathbf{n}^{V}, \Gamma_{L}\right)-\sum_{i=1}^{M} \mu_{i}^{\mathrm{tot}}\left(\int_{\Gamma_{L}} n_{i}(r) \psi(r) d r+\int_{\Gamma_{V}} n_{i}(r) \psi(r) d r-\frac{N_{i}^{\mathrm{tot}}}{V_{0}}\right) \\
= & \phi\left(S_{L} f_{b}\left(\mathbf{n}^{L}\right)+S_{V} f_{b}\left(\mathbf{n}^{V}\right)\right)+\sigma_{s l} \int_{\Gamma_{L}} A_{s}(r) \psi(r) d r+\sigma_{s v} \int_{\Gamma_{V}} A_{s}(r) \psi(r) d r \\
& -\phi \sum_{i=1}^{M} \mu_{i}^{\mathrm{tot}}\left(S_{L} n_{i}^{L}+S_{V} n_{i}^{V}\right)+\sum_{i=1}^{M} \frac{\mu_{i}^{\mathrm{tot}} N_{i}^{\mathrm{tot}}}{V_{0}} .
\end{aligned}
$$

Setting the partial derivative of $L_{f}$ with respect to $\mu_{i}^{\text {tot }}$ to zero, we quickly recover the conservation constraint for species $i$. It is also easy to get its partial derivative with respect to the liquid phase molar concentration:

$$
\frac{\partial L_{f}}{\partial n_{i}^{L}}=\phi S_{L} \mu_{i}^{L}-\phi S_{L} \mu_{i}^{\text {tot }}
$$

The stationarity condition $\frac{\partial L_{f}}{\partial n_{i}^{L}}=0$ implies $\mu_{i}^{L}=\mu_{i}^{\text {tot }}$. Similarly, we have

$$
\frac{\partial L_{f}}{\partial n_{i}^{V}}=\phi S_{V} \mu_{i}^{V}-\phi S_{V} \mu_{i}^{\mathrm{tot}}
$$

and the corresponding stationarity condition $\frac{\partial L_{f}}{\partial n_{i}^{V}}=0$ implies $\mu_{i}^{V}=\mu_{i}^{\text {tot }}$. These two stationarity conditions together imply $\mu_{i}^{L}=\mu_{i}^{V}$, a thermal equilibrium property we expect.

Since $A_{s}(r)$ is a monotonic function of $r$, it makes sense to assume that both domains $\Gamma_{L}$ and $\Gamma_{V}$ are simply two intervals. Without loss of generality, we assume that the rock (the porous medium we consider) is liquid wetting, i.e. $\sigma_{s l}<\sigma_{s v}$. In this case, the liquid phase would like to occupy small pores while gas phase would like to occupy large pores. We denote the borderline pore size separating $\Gamma_{L}$ and $\Gamma_{V}$ by $R_{\text {intf }}$. We assume $\Gamma=\left[R_{\min }, R_{\max }\right]$, we then have $\Gamma_{L}=\left[R_{\min }, R_{\text {intf }}\right)$ and $\Gamma_{V}=\left[R_{\text {intf }}, R_{\max }\right]$. Then the Lagrangian function becomes

$$
\begin{aligned}
& L_{f}\left(\mathbf{n}^{L}, \mathbf{n}^{V}, R_{\text {intf }}, \boldsymbol{\mu}^{\text {tot }}\right) \\
:= & f^{\mathrm{tot}}\left(\mathbf{n}^{L}, \mathbf{n}^{V}, R_{\text {intf }}\right)-\sum_{i=1}^{M} \mu_{i}^{\mathrm{tot}}\left(\int_{\Gamma_{L}} n_{i}(r) \psi(r) d r+\int_{\Gamma_{V}} n_{i}(r) \psi(r) d r-\frac{N_{i}^{\mathrm{tot}}}{V_{0}}\right) \\
= & \phi\left(S_{L} f_{b}^{L}+S_{V} f_{b}^{V}\right)+\sigma_{s l} \int_{R_{\text {min }}}^{R_{\text {intf }}} A_{s}(r) \psi(r) d r+\sigma_{s v} \int_{R_{\text {intf }}}^{R_{\text {max }}} A_{s}(r) \psi(r) d r \\
& -\phi \sum_{i=1}^{M} \mu_{i}^{\text {tot }}\left(S_{L} n_{i}^{L}+S_{V} n_{i}^{V}\right)+\sum_{i=1}^{M} \frac{\mu_{i}^{\text {tot }} N_{i}^{\text {tot }}}{V_{0}},
\end{aligned}
$$


where $f_{b}^{L}:=f_{b}\left(\mathbf{n}^{L}\right)$ and $f_{b}^{V}:=f_{b}\left(\mathbf{n}^{V}\right)$.

The derivative of the Lagrangian function with respect to $R_{\text {intf }}$ is

$$
\begin{aligned}
\frac{\partial L_{f}}{\partial R_{\text {intf }}}= & \phi f_{b}^{L} \frac{\partial S_{L}}{\partial R_{\text {intf }}}+\phi f_{b}^{V} \frac{\partial S_{V}}{\partial R_{\text {intf }}}+\left(\sigma_{s l}-\sigma_{s v}\right) A_{s}\left(R_{\text {intf }}\right) \psi\left(R_{\text {intf }}\right) \\
& -\phi \sum_{i=1}^{M} \mu_{i}^{\text {tot }} n_{i}^{L} \frac{\partial S_{L}}{\partial R_{\text {intf }}}-\phi \sum_{i=1}^{M} \mu_{i}^{\text {tot }} n_{i}^{V} \frac{\partial S_{V}}{\partial R_{\text {intf }}}
\end{aligned}
$$

We note that

$$
\frac{\partial S_{V}}{\partial R_{\text {intf }}}=\frac{\partial}{\partial R_{\text {intf }}}\left(\frac{\int_{R_{\text {intf }}}^{R_{\max }} \psi(r) d r}{\int_{R_{\min }}^{R_{\max }} \psi(r) d r}\right)=-\frac{\psi\left(R_{\mathrm{intf}}\right)}{\phi},
$$

and similarly

$$
\frac{\partial S_{L}}{\partial R_{\text {intf }}}=\frac{\psi\left(R_{\text {intf }}\right)}{\phi}
$$

Using the fact that

$$
p^{L}=\sum_{i=1}^{M} \mu_{i}^{L} n_{i}^{L}-f_{b}^{L}=\sum_{i=1}^{M} \mu_{i}^{\mathrm{tot}} n_{i}^{L}-f_{b}^{L},
$$

and

$$
p^{V}=\sum_{i=1}^{M} \mu_{i}^{V} n_{i}^{V}-f_{b}^{V}=\sum_{i=1}^{M} \mu_{i}^{\mathrm{tot}} n_{i}^{V}-f_{b}^{V},
$$

we conclude that

$$
\begin{aligned}
\frac{\partial L_{f}}{\partial R_{\text {intf }}}= & \psi\left(R_{\text {intf }}\right)\left(f_{b}^{L}-f_{b}^{V}-\sum_{i=1}^{M} \mu_{i}^{\text {tot }} n_{i}^{L}+\sum_{i=1}^{M} \mu_{i}^{\text {tot }} n_{i}^{V}\right) \\
& +\left(\sigma_{s l}-\sigma_{s v}\right) A_{s}\left(R_{\text {intf }}\right) \psi\left(R_{\text {intf }}\right) \\
= & \psi\left(R_{\text {intf }}\right)\left(p^{V}-p^{L}\right)+\left(\sigma_{s l}-\sigma_{s v}\right) A_{s}\left(R_{\text {intf }}\right) \psi\left(R_{\text {intf }}\right)
\end{aligned}
$$

Since $\psi\left(R_{\text {intf }}\right) \neq 0$, the stationarity condition $\frac{\partial L_{f}}{\partial R_{\text {intf }}}=0$ yields

$$
\Delta p:=p^{V}-p^{L}=\left(\sigma_{s v}-\sigma_{s l}\right) A_{s}\left(R_{\text {intf }}\right) .
$$

Finally, we recall (9), and conclude

$$
\Delta p=\frac{2\left(\sigma_{s v}-\sigma_{s l}\right)}{R_{\text {intf }}} .
$$

Clearly, for a liquid-wetting system, $\Delta p:=p^{V}-p^{L}>0$ as expected. 
If the system is gas-wetting, we will have a similar derivation and end up the same equations (16) and (17).

To see our result (17) is consistent with the classical Young-Laplace equation, we consider a sufficiently narrow (so that we can ignore gravity) tube of circular cross-section (radius $\left.R_{\text {intf }}\right)$, where the interface between two fluids forms a meniscus that is a portion of the surface of a sphere with radius $R_{\text {curv }}$. The pressure jump across this surface is related to the radius and the surface tension $\sigma_{l v}$ by

$$
\Delta p=\frac{2 \sigma_{l v}}{R_{\text {curv }}}
$$

The radius of the sphere will be a function only of the contact angle, $\theta_{\text {contact }}$, which in turn depends on the exact properties of the fluids and the solids in which they are in contact:

$$
R_{\text {curv }}=\frac{R_{\text {intf }}}{\cos \theta_{\text {contact }}}
$$

We thus have

$$
\Delta p=\frac{2 \sigma_{l v} \cos \theta_{\text {contact }}}{R_{\text {intf }}}
$$

which is known as the Young-Laplace equation.

Our derived equation (17) is exactly the same as the Young-Laplace equation, because it is well known that at equilbirum, due to the force balance along the tangential direction of the wall, we have

$$
\sigma_{s v}-\sigma_{s l}=\sigma_{l v} \cos \theta_{\text {contact }} .
$$

We would like to remark on the role of the liquid-vapor interfacial tension $\sigma_{l v}$ in Darcyscale modeling of phase equilibrium. This liquid-vapor interfacial tension $\sigma_{l v}$, together with $\sigma_{s v}$ and $\sigma_{s l}$ affects the contact angle and the shape of the meniscus at the pore scale. However, it is quite surprising to see that the liquid-vapor interfacial tension $\sigma_{l v}$ does not affect substantially the degree of capillarity at Darcy's scale. As we see from the above analysis, the Darcy-scale capillarity is completely determined by the difference between $\sigma_{s v}$ and $\sigma_{s l}$ in our simplified model. In particular, if $\sigma_{s v}$ and $\sigma_{s l}$ are the same, we do not have any capillary pressure, no matter how larger the liquid-vapor interfacial tension $\sigma_{l v}$ is.

\subsection{Compositional grading}

Composition of a fluid mixture is usually considered to be homogeneous at a lab scale within its single-phase region, but in a geological scale, composition of components has been observed to vary vertically. The are a number of reasons why composition of reservoir fluid mixture varies with depth, but most people agree that gravity is the major cause, as it segregates the heaviest oil components toward the bottom of the reservoir and lighter components like methane toward the top. Most of existing modeling approaches of compositional grading in literature does not consider pore size distribution, which is what we intent to simulate, a novel feature of our model. 
In the modeling of compositional grading, people are primarily interested in the variation in the depth $z$ direction only. For convenience, we now also consider only one spatial dimension in the vertical direction $z$; that is, the position vector $\mathbf{x}$ is reduced to $z$. The more general problem in three spatial dimensions now reduces to a one-dimensional problem in space (together with another dimension in pore size). We still ignore $\sigma_{l v}$. We take positive $z$ direction pointing downward to the center of earth, and we denote $g=|\mathbf{g}|$. We consider a 1-D domain of $[z=0, z=H]$ and physically the cross-sectional area can be viewed as $1 \times 1$ meter $^{2}$. We assume $\Gamma=\left[R_{\min }, R_{\max }\right]$. The total Helmholtz free energy has the following form

$$
F^{\mathrm{tot}}[\mathbf{n}]=\int_{0}^{H} \int_{R_{\min }}^{R_{\max }}\left(f_{b}(\mathbf{n}(z, r))+A_{s}(r) \sigma_{s f}(\mathbf{n}(z, r))-g z \sum_{i=1}^{M} M_{w, i} n_{i}(z, r)\right) \psi(z, r) d r d z
$$

Using the same variational approach as we discussed above, we can obtain the following stationarity condition

$$
\mu_{i}(\mathbf{n}(z, r))+A_{s}(r) \mu_{i}^{\sigma}(\mathbf{n}(\mathbf{x}, r))-g z M_{w, i}=\mu_{i}^{\mathrm{tot}} .
$$

The equation (18) can be used to solve the molar concentration $\mathbf{n}$ as a function of $z$ and $r$, which is our primary unknown. Obviously, closed-form analytical solutions unlikely exist for $(18)$, and thus we need to find its numerical solution. Once the molar concentration $\mathbf{n}$ is obtained, we can quickly recover pressure distribution (as a function of depth $z$ and pore radius $r$ ) as well as chemical potential and various types of free energies.

\subsection{Hydrostatic equilibrium}

In fluid mechanics, when a fluid is at rest, or when the flow velocity at each point of the fluid body is zero, we say that the fluid is in hydrostatic equilibrium. Hydrostatic equilibrium in a partially saturated porous medium has richer behaviors than it in a free space; in particular the interplay of gravity and capillary force yields a variation of fluid saturation in the depth direction. Our model above can be used to model and predict such variation of fluid saturation in the depth direction.

For simplicity, we now consider a single-component two-phase system, still in onedimensional domain but with a homogeneous porous medium. Gravity and capillarity are modeled, and we allow a certain pore size distribution, which is independent of depth. The total Helmholtz free energy has the following form

$$
F^{\mathrm{tot}}[n]=\int_{0}^{H} \int_{R_{\min }}^{R_{\max }}\left(f_{b}(n(z, r))+A_{s}(r) \sigma_{s f}(n(z, r))-g z M_{w} n(z, r)\right) \psi(r) d r d z
$$

The stationarity condition becomes

$$
\mu(n(z, r))+A_{s}(r) \mu^{\sigma}(n(z, r))-g z M_{w}=\mu^{\text {tot }} .
$$

For simplicity, we further assume $\mu^{\sigma}(n(z, r))$ is a constant, which could be justified in certain scenarios (for example, if we take the linear model for $\sigma_{s f}(n)$, then $\mu^{\sigma}(n(z, r)$ ) is 
indeed a constant). In this case, solution of $(19)$ can be reduced further if the boundary condition also matches. We first solve the following problem numerically,

$$
\mu\left(n_{R}(\lambda)\right)+\lambda=\mu^{\text {tot }}
$$

with certain boundary condition consistent to the one of the original problem. After obtaining the reduced solution $n_{R}$, the solution to the original problem (19) can be recovered by

$$
n(z, r)=n_{R}\left(A_{s}(r) \mu^{\sigma}-g z M_{w}\right) .
$$

We note that this reduction of dimenions works only for single-compoennt systems. For systems consisting of two or more components, the indepedent variable $r$ and $z$ cannot be reduced as the way of gravity to redistribute species might differ from the affect of capillarity to redistribute species, because the former is molecular weight-dependent while the latter is independent of molecular weights.

\section{Energy-stable numerical schemes based on convex-concave splitting}

To get a numerical solution to (14), we first convert it to the following time-dependent equation

$$
\frac{\partial n_{i}}{\partial t}=\nabla_{(\mathbf{x}, r)} D_{i} \nabla_{(\mathbf{x}, r)}\left(\mu_{i}(\mathbf{n}(\mathbf{x}, r))+A_{s}(r) \mu_{i}^{\sigma}(\mathbf{n}(\mathbf{x}, r))-(\mathbf{g} \cdot \mathbf{x}) M_{w, i}\right),
$$

where $D_{i}$ is the effective diffusion coefficient for species $i$. In the following discussion, we will propose an energy-stable numerical scheme based on convex-concave splitting to solve (21). We assume that when time goes infinity, the solution to (21) will converge to an equilbrium solution, which should be a solution to (14). The time-marching solution to (21) can be then viewed as an iterative solution procedure to (14). On the other hand, (21) can be also used to simulate the dynamics of fluid mixture in reality, provided that the bulk flow is small and negligible, and the diffusion is dominant with a diagonal diffusion tensor used above.

In this section, we first review the fact that the total Helmholtz free energy density can be split into the summation of two parts: one is convex and the other is concave, even though this type convex-concave splitting is not unique. We then apply an implicit time marching scheme for the convex part and an explicit scheme for the concave part, which can be shown to yield always an stable and energy-dissipated semi-implicit time marching scheme that is often also computationally efficient.

We first note that the gravity term $(\mathbf{g} \cdot \mathbf{x}) M_{w, i}$ is always linear with respect to $\mathbf{x}$ (and constant with respective to $r$ ), and thus we can safely treat it using an explicit scheme. We now consider the fluid-solid interaction term. For simplicity, we assume $\mu_{i}^{\sigma}(\mathbf{n}(\mathbf{x}, r))=\mu_{i}^{\sigma}$ is a constant. Under this assumption, the term $A_{s}(r) \mu_{i}^{\sigma}(\mathbf{n}(\mathbf{x}, r))$ can also be safely treated using an explicit scheme. We now look at the bulk term $\mu_{i}(\mathbf{n})$, which is the most challenging term to treat numerically.

It is well known that the ideal contribution of the bulk Helmholtz free energy density is convex with respect to molar concentrations. In fact, the fluid mixture may split into 
multiple phases if it is not convex [4, 5, 27]. We note that the repulsion force results is a convex contribution to the bulk Helmholtz free energy density [27] for single-component fluid, while a non-convex contribution [4] for multi-component mixtures $(M \geq 2)$. We also note that for the pure substance, the attraction term in the bulk Helmholtz free energy density from Peng-Robinson equation of state is proved to result in a concave contribution [27. Unfortunately, again it fails to remain so for multi-component mixture. In this paper, we adopt the splitting originally proposed in [12], where an additional ideal term is used to construct a strict convex-concave splitting for multi-component mixture. We first choose an algorithm-turning parameter $\lambda>0$ and then split the bulk chemical potential calculation as

$$
\mu_{i}(\mathbf{n})=\mu_{i}^{\text {convex }}(\mathbf{n})+\mu_{i}^{\text {concave }}(\mathbf{n}) .
$$

Similarly, the bulk Helmholtz free energy density is also split as

$$
f_{b}(\mathbf{n})=f_{b}^{\text {convex }}(\mathbf{n})+f_{b}^{\text {concave }}(\mathbf{n}),
$$

where

$$
\begin{gathered}
f_{b}^{\text {convex }}(\mathbf{n})=(1+\lambda) f_{b}^{\text {ideal }}(\mathbf{n})+f_{b}^{\text {repulsion }}(\mathbf{n}), \\
f_{b}^{\text {concave }}(\mathbf{n})=f_{b}^{\text {attraction }}(\mathbf{n})-\lambda f_{b}^{\text {ideal }}(\mathbf{n})
\end{gathered}
$$

We remark that with a sufficiently large $\lambda$, we can always obtain a strict convex-concave splitting for the bulk Helmholtz free energy density of multi-component mixture. In practical computations, taking $\lambda=0.1$ is sufficient for most numerical cases.

With this convex-concave splitting, our energy-stable time marching scheme can be formulated as

$$
\frac{n_{i}^{k+1}-n_{i}^{k}}{t^{k+1}-t^{k}}=\nabla_{(\mathbf{x}, r)} D_{i} \nabla_{(\mathbf{x}, r)}\left(\mu_{i}^{\text {convex }}\left(\mathbf{n}^{k+1}\right)+\mu_{i}^{\text {concave }}\left(\mathbf{n}^{k}\right)+A_{s}(r) \mu_{i}^{\sigma}-(\mathbf{g} \cdot \mathbf{x}) M_{w, i}\right)
$$

We can approximate this equation further using a spatial discretization method. We recommend the cell-center finite difference method, the stagger-grid finite volume method, and the mixed finite element methods. In this paper, we use the cell-center finite difference method. Due to the page limitation, we skip the numerical implementation of our model.

\section{Numerical examples}

\subsection{Compositional grading of a binary mixture}

In the first example, we consider a binary mixture of carbon dioxide $\left(\mathrm{CO}_{2}\right)$ and normal decane $\left(\mathrm{nC}_{10}\right)$. We consider a one-dimensional domain along the gravity (vertical) direction with the height of 200 meters. We use a uniform mesh with 100 cells. We do not consider a general pore size distribution in this example, and we assume all pores in the medium have a size $r=10^{-6}$ meters. At the initial time, carbon dioxide and normal decane distribute 


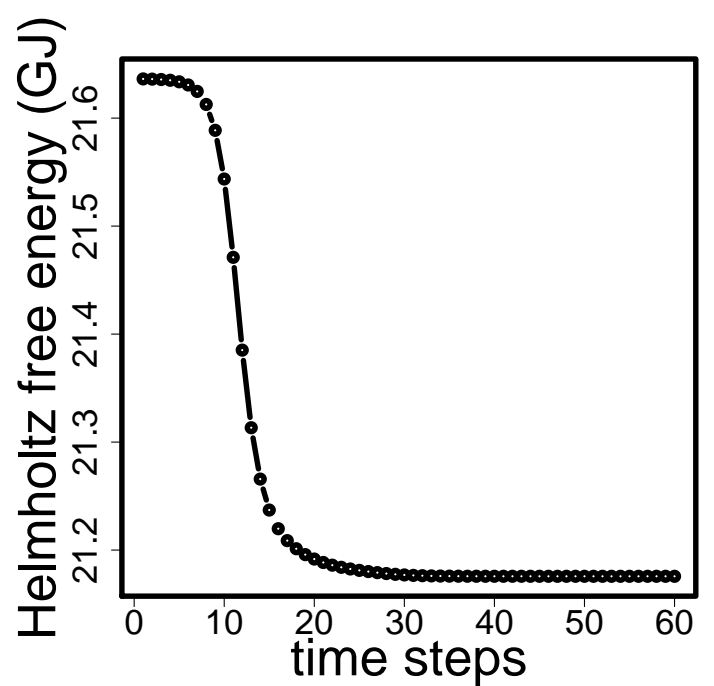

(a) convergence history of entire 60 time steps

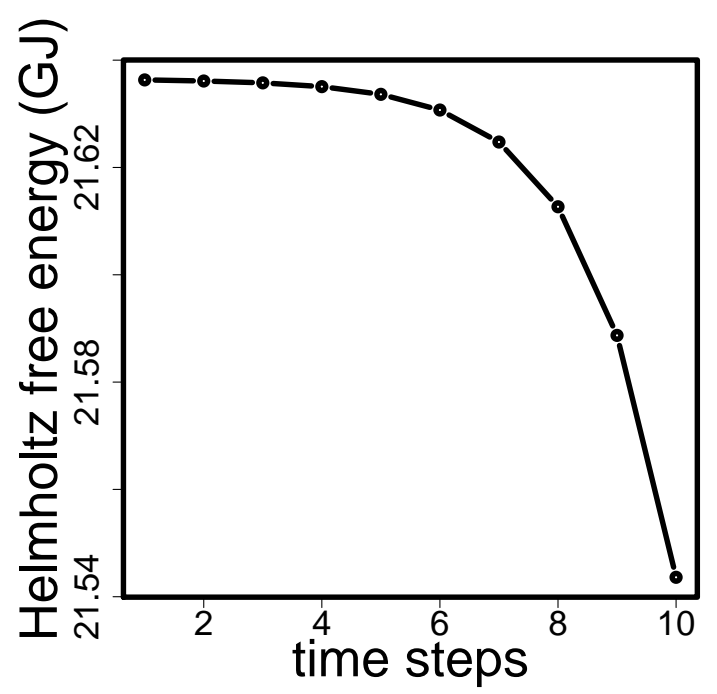

(b) convergence history of first 10 time steps

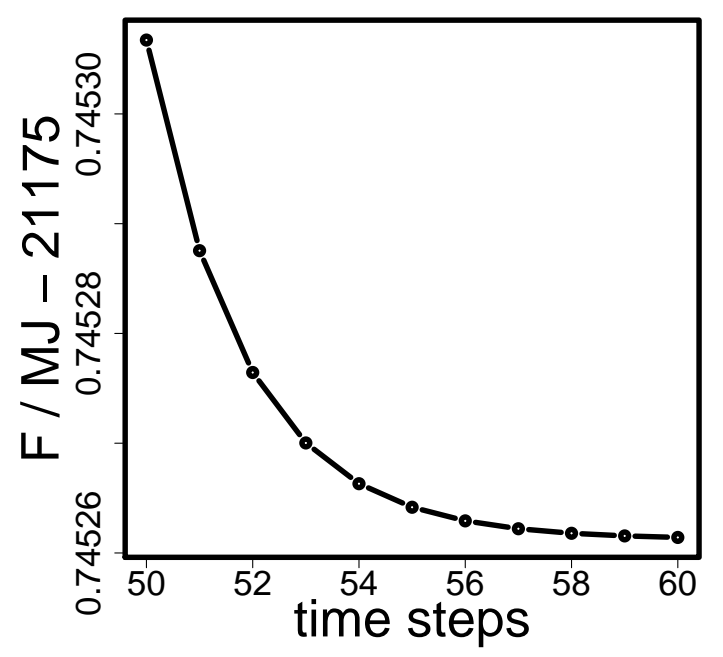

(c) convergence history of final 10 time steps

Figure 1: Energy dissipation with time steps for the binary system of $\mathrm{CO}_{2}$ and $\mathrm{nC}_{10}$ in Example 1. 


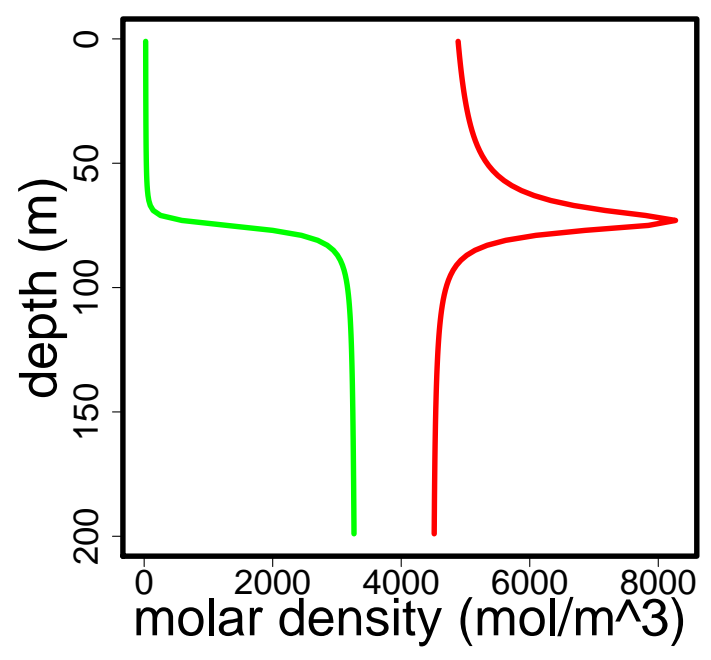

(a) at time step 20

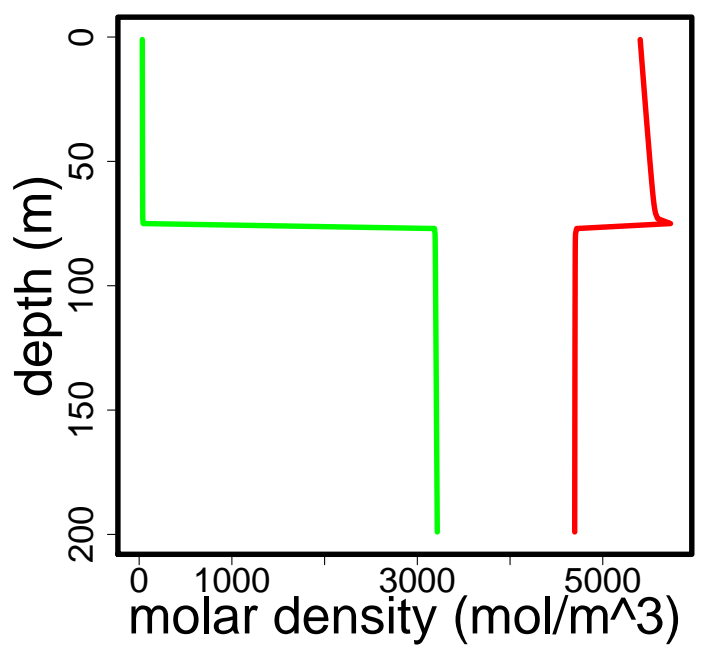

(c) at time step 40

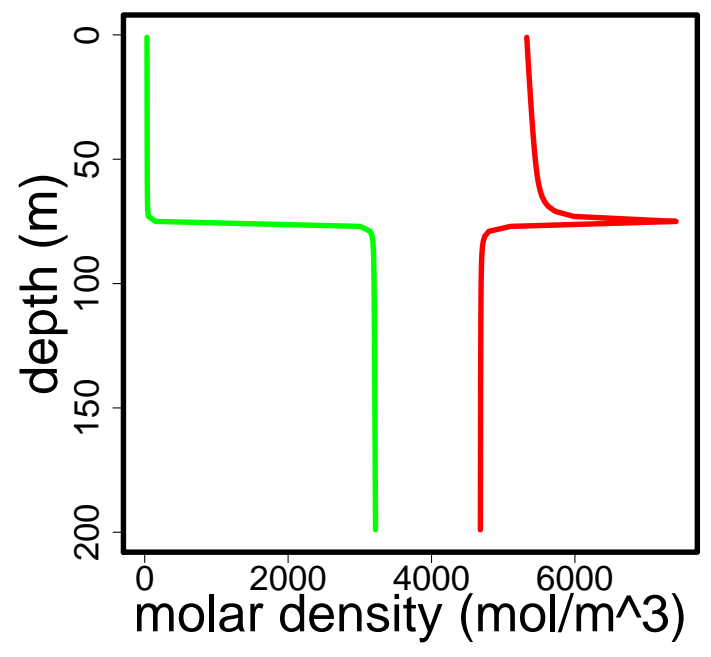

(b) at time step 30

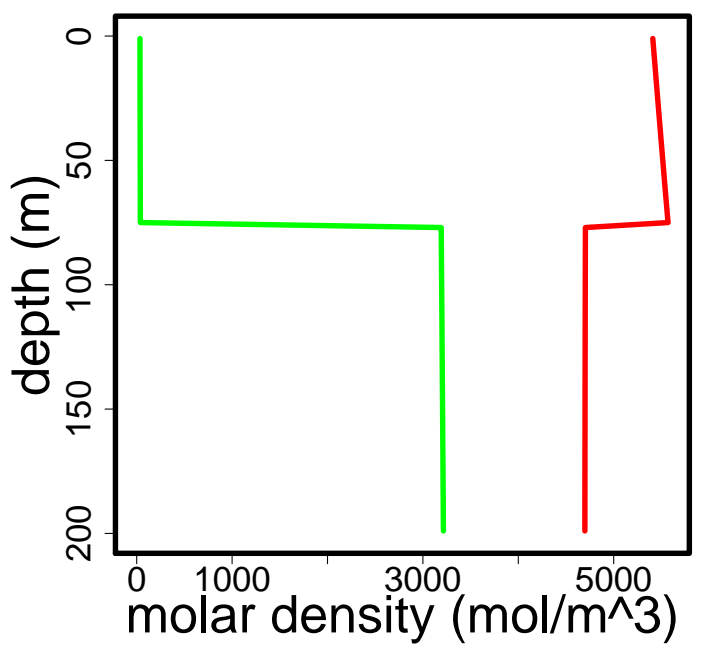

(d) at time step 60

Figure 2: Molar concentration of $\mathrm{CO}_{2}$ (red lines) and $\mathrm{nC}_{10}$ (blue lines) at various time steps in Example 1. 


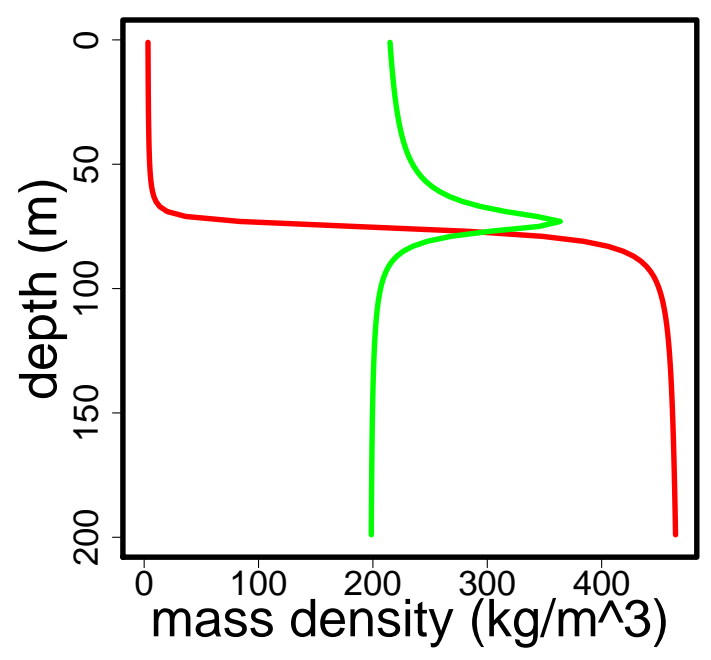

(a) at time step 20

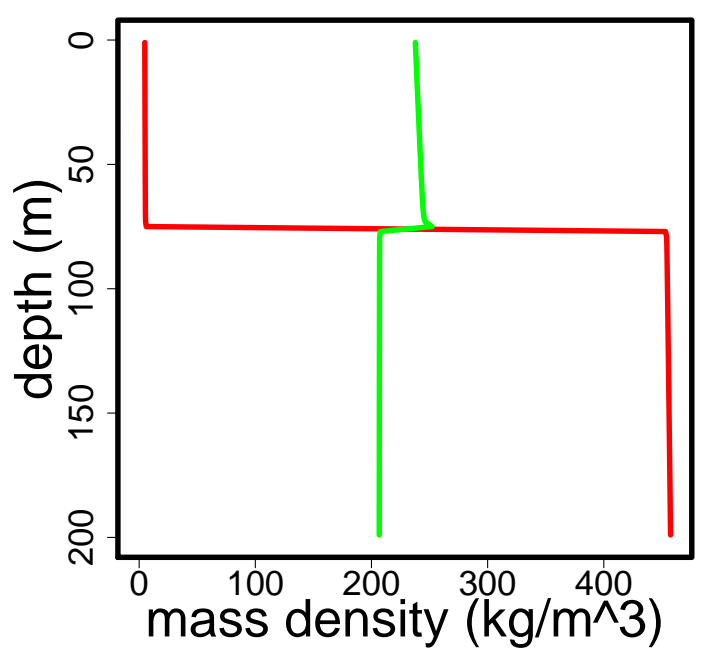

(c) at time step 40

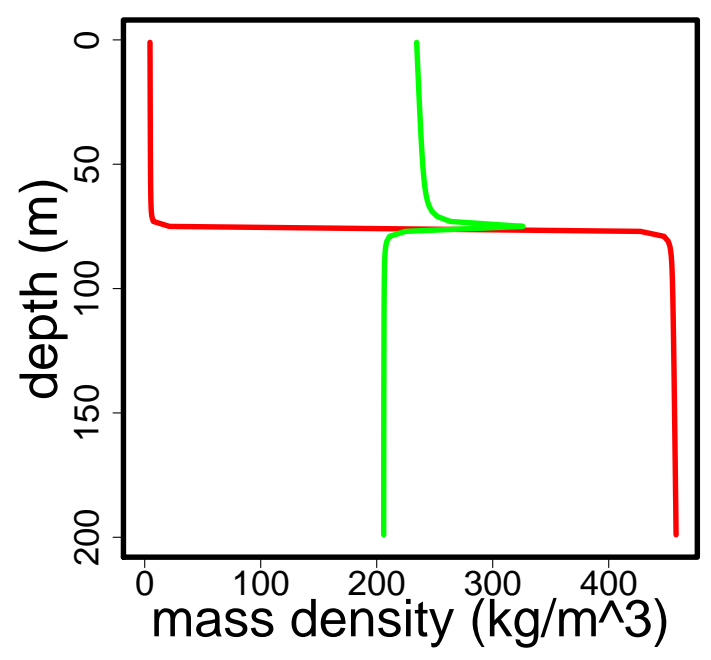

(b) at time step 30

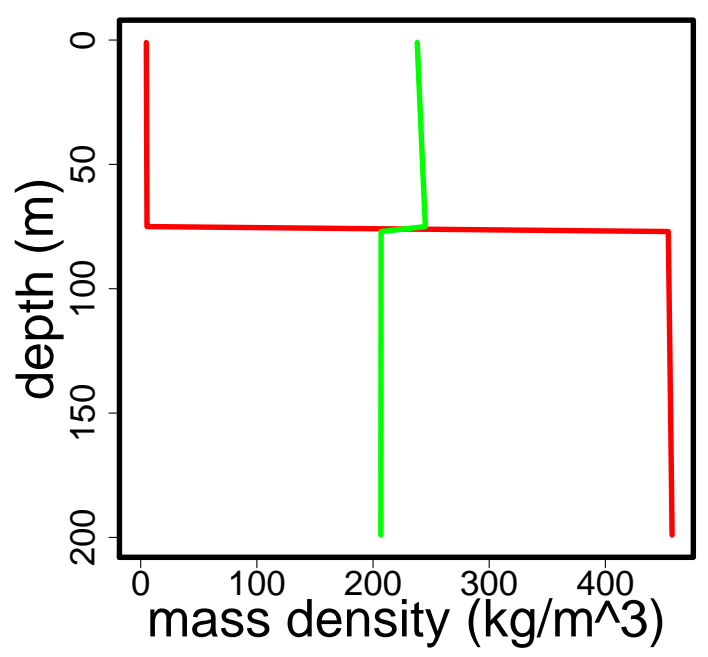

(d) at time step 60

Figure 3: Mass concentration of $\mathrm{CO}_{2}$ (red lines) and $\mathrm{nC}_{10}$ (blue lines) at various time steps in Example 1. 


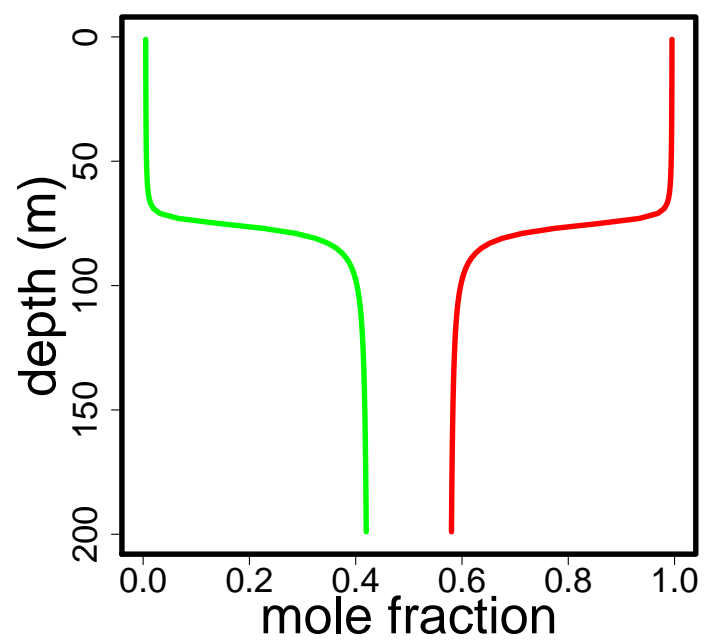

(a) at time step 20

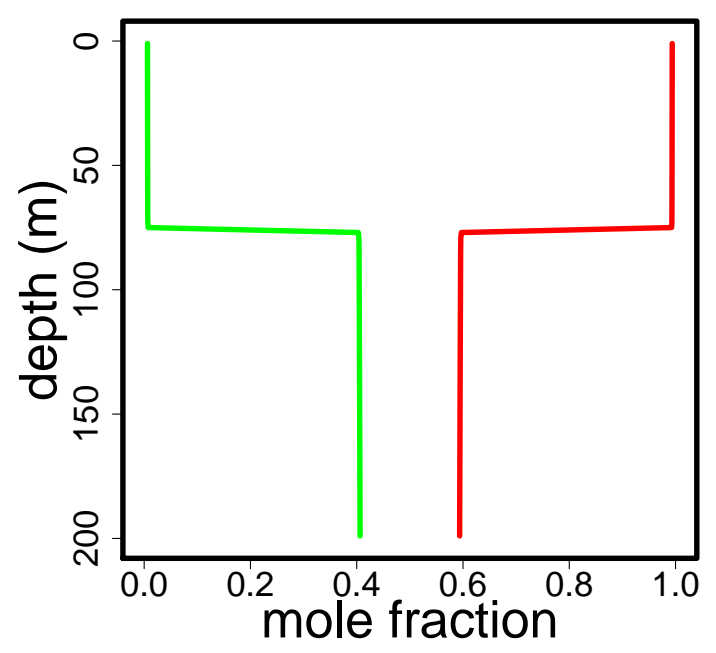

(c) at time step 40

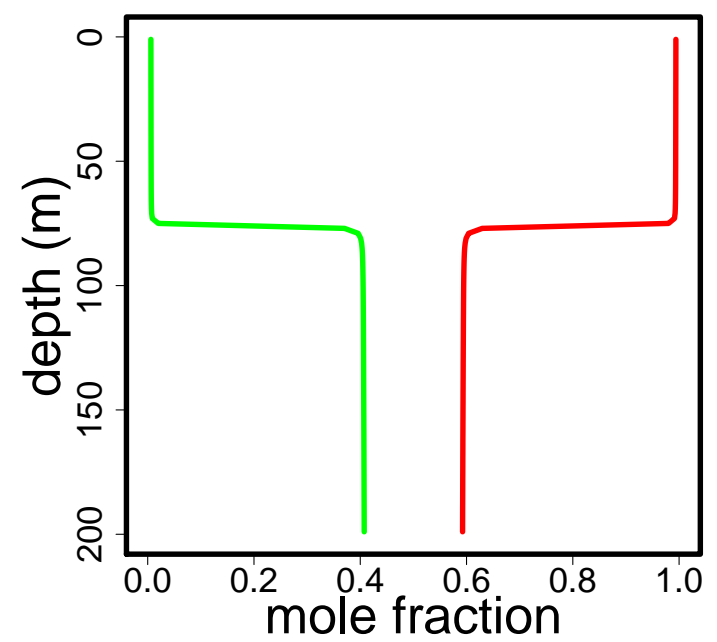

(b) at time step 30

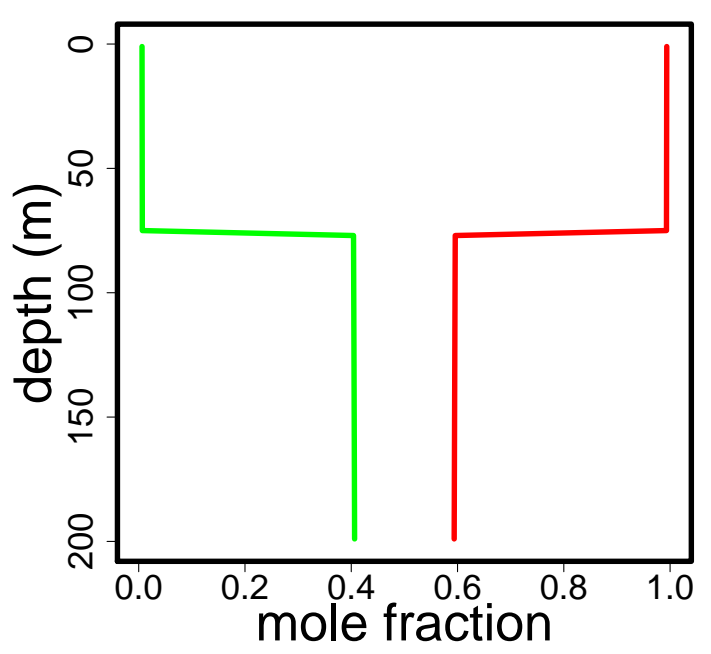

(d) at time step 60

Figure 4: Mole fraction of $\mathrm{CO}_{2}$ (red lines) and $\mathrm{nC}_{10}$ (blue lines) at various time steps in Example 1. 
uniformly in the space (in terms of their molar concentration). We impose the initial molar concentrations of $5000 \mathrm{~mol} / \mathrm{m}^{3}$ and $2000 \mathrm{~mol} / \mathrm{m}^{3}$ for carbon dioxide and normal decane respectively.

The convergence history of the time marching scheme for the total Helmholtz free energy is shown in Figure 1(a). Clearly, the computed total Helmholtz free energy decays with time steps as our theory predicts. The decay of calculated free energy ensures the numerical stability of our scheme. In Figure 1(a), one observes that the energy decay is slow initially in the first 5 time steps, and then the energy starts to decay rapidly, in particular from time step 10 to time step 15. After time step 20, the decay quickly slows down when the solution starts to approach its steady state. It is evident that the fast decay period corresponds to the phase splitting, which is the cause of the fast decay during that time period. After phase splitting is carried out, the species slowly redistribute themselves further driven by gravity, which slightly decreases the total Helmholtz free energy further in later time steps. To indicate that the total Helmholtz free energy indeed decreases even in early time steps and later time steps, we also depict the early and later convergence history in Figure 1(b) and Figure 1(c).

Spatial variation of molar concentration is displayed in Figure 2(a)-2(d) for each species at various time steps. It is clear that phase splitting occurred while the system lowers down its total Helmholtz free energy. As expected, the light component $\left(\mathrm{CO}_{2}\right)$ segregates toward the top while the heavy component $\left(\mathrm{nC}_{10}\right)$ concentrates toward the bottom. In early time steps, the molar concentration profiles are smooth functions of depth. When the system reaches its steady state (equilibrium state), there is a discontinuity in molar concentration of each species. The molar density (summation of the molar concentration) also has a jump across the liquid-vapor interface. Since the depth of the reservoir simulated here is only 200 meters, the molar density variation with depth in each of the two single-phase regions alone is not significant. Among them, the relatively pronounced variation is the change of $\mathrm{CO}_{2}$ molar concentration with depth in the gas phase region.

As mass concentration usually does a better job to show the difference of segregation between species of different molecular weights, we also provide the spatial variation of mass concentration in Figure 3(a)-3(d). It is interesting to observe that the mass concentration of $\mathrm{CO}_{2}$ in gas phase is only slightly larger than it in the oil phase; this is because the significant solubility of $\mathrm{CO}_{2}$ in oil. Finally, we also plot the mole fraction of each species as a function of depth at various time steps. We observe again the discontinuity of computed variables at later times steps; that is, the mole fraction changes with depth quite continuously in early time steps, but a jump eventually forms when it reaches its equilibrium state.

\subsection{Competition between capillary and gravity forces}

In the second example, we simulate a binary mixture of methane $\left(\mathrm{C}_{1}\right)$ and normal decane $\left(\mathrm{nC}_{10}\right)$. We again consider a one-dimensional domain along the gravity (vertical) direction with the height of 10 meters, discretized using a uniform mesh with 100 cells. We consider both gravity and capillarity. At the initial time, we put into the porous medium methane and normal decane with uniform distribution of molar concentration in the space. The 

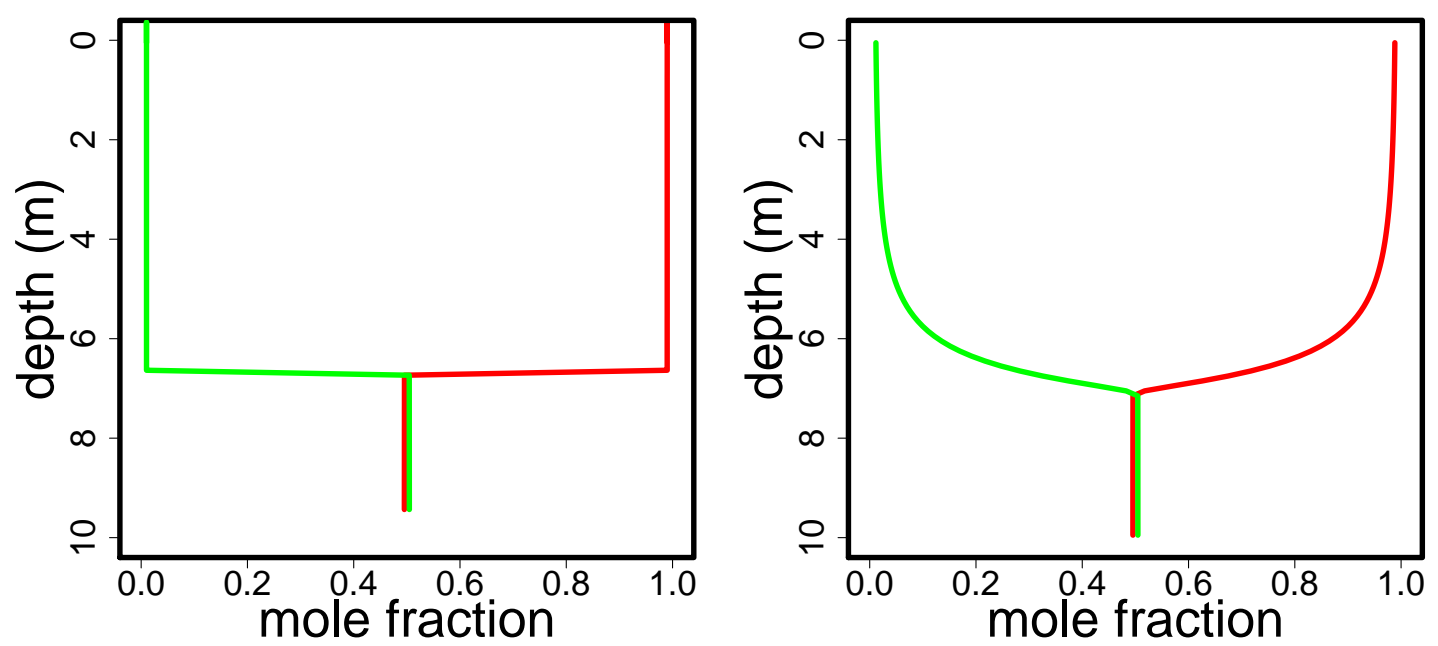

(a) monodisperse pore-size distribution (case I)

(b) lognormal pore-size distribution (case II)

Figure 5: Mole fraction of $\mathrm{C}_{1}$ (red lines) and $\mathrm{nC}_{10}$ (blue lines) in Example 2.
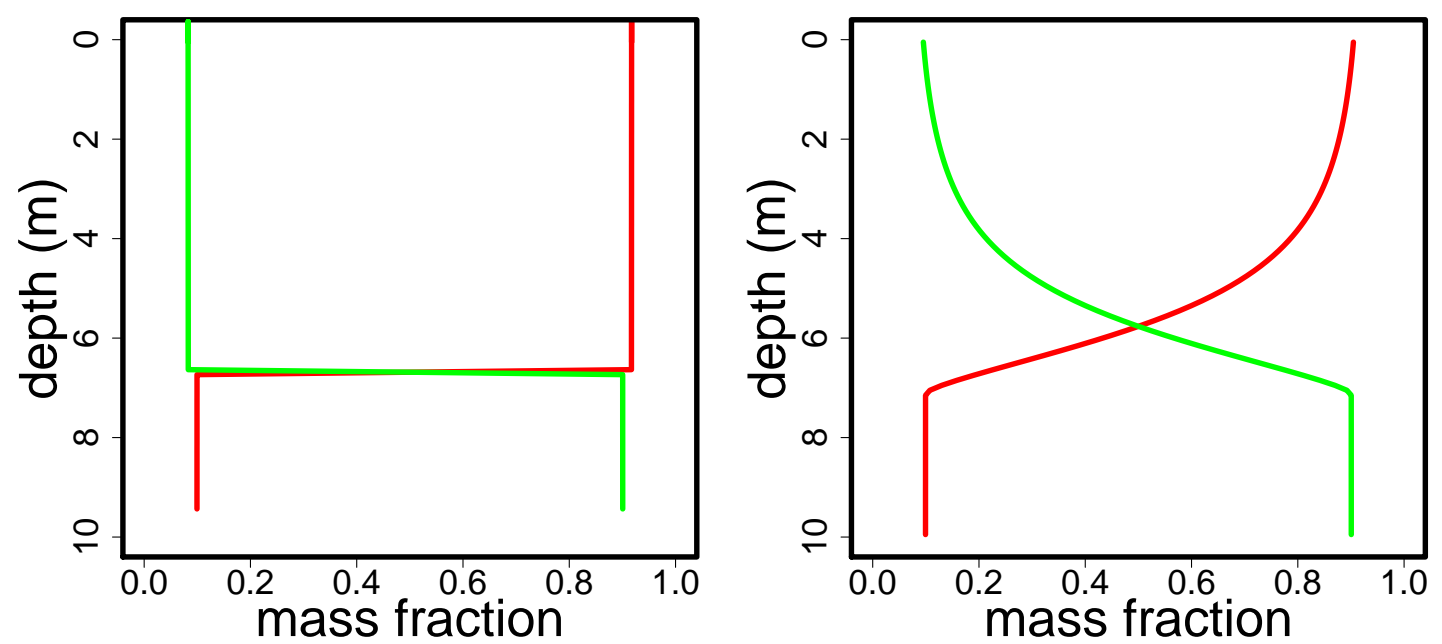

(a) monodisperse pore-size distribution (case I) (b) lognormal pore-size distribution (case II)

Figure 6: Mass fraction of $\mathrm{C}_{1}$ (red lines) and $\mathrm{nC}_{10}$ (blue lines) in Example 2. 

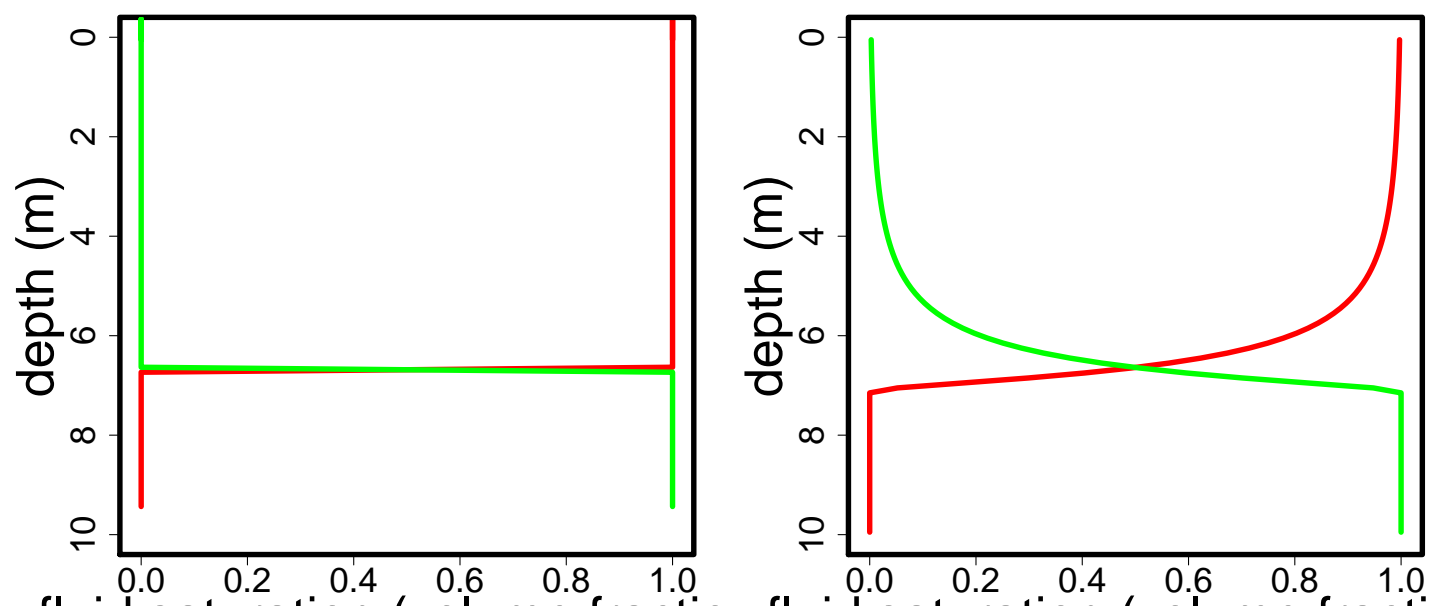

fluid saturation (volume fraction fluid saturation (volume fractio

(a) monodisperse pore-size distribution (case I) $\quad$ (b) lognormal pore-size distribution (case II)

Figure 7: Saturation of the $\mathrm{C}_{1}$-rich phase (red lines) and the $\mathrm{nC}_{10}$-rich phase (blue lines) in Example 2.

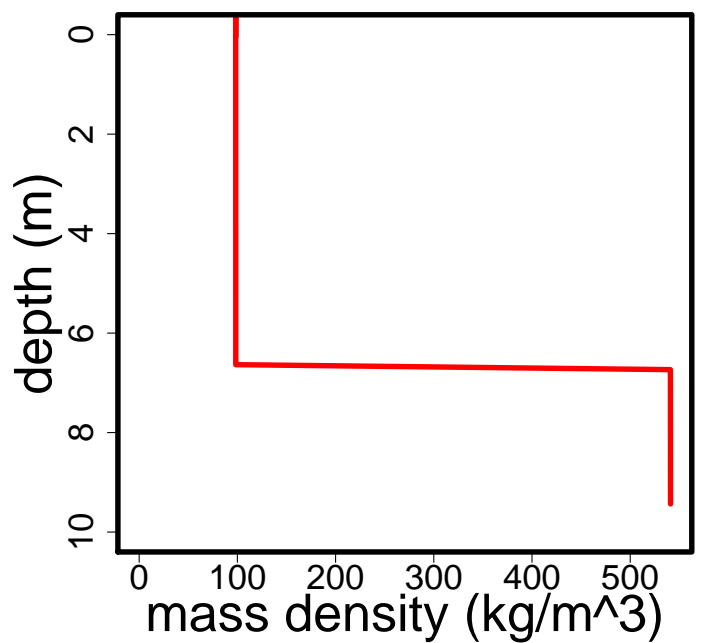

(a) monodisperse pore-size distribution (case I)

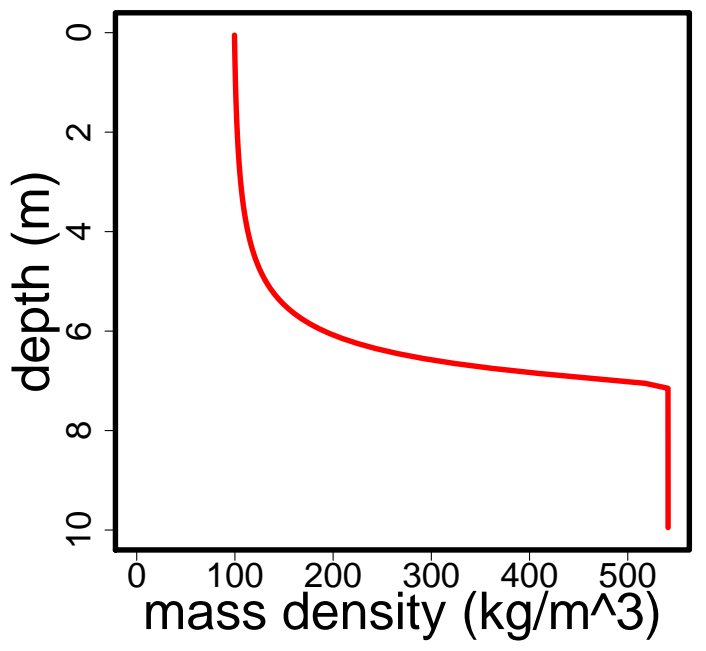

(b) lognormal pore-size distribution (case II)

Figure 8: Overall mass density of the fluid mixture in Example 2. 


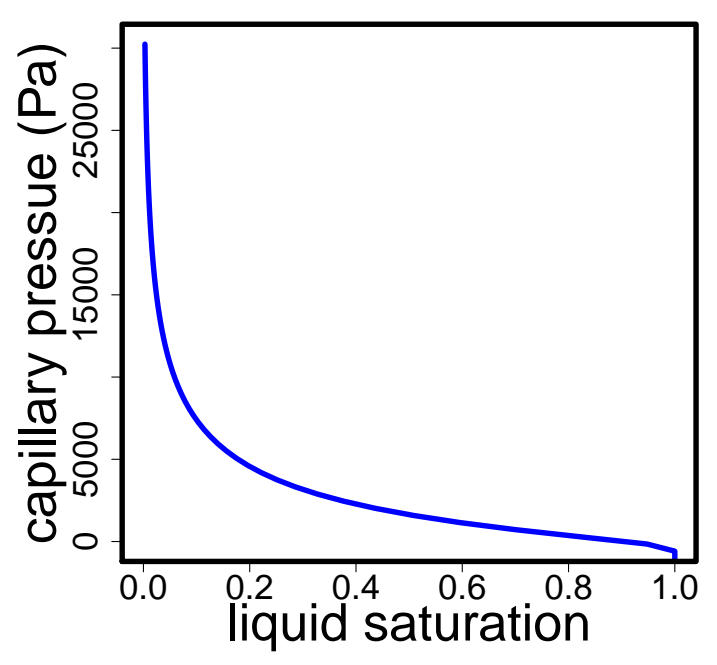

Figure 9: Capillary pressure as a function of saturation computed in Example 2 (case II).

initial molar concentrations of $5000 \mathrm{~mol} / \mathrm{m}^{3}$ and $2000 \mathrm{~mol} / \mathrm{m}^{3}$ are imposed for methane and normal decane respectively.

In the test, we consider two cases. In Case I, we assume that all pores have one identical size of $10^{-6}$ meters, while in Case II, we assume that the pore size $r$ follows the log-normal distribution. The mean of $\log (r /$ meter $)$ is $\log \left(10^{-6}\right)$ and the standard deviation of is 1 . In both cases, we assume $\mu^{\sigma}=-2 \times 10^{-7} \mathrm{~J} \cdot \mathrm{m} / \mathrm{mol}$. A negative value of $\mu^{\sigma}$ represents liquid-wetting rock.

Spatial variation of mole fraction of each species is displayed in Figure 5(a)-5(b) for both the case I and case II. Clearly, the mole fraction has a jump between the liquid-phase and vapor-phase regions in Case I of monodisperse pore-size distribution. In case II, pore size has a spread distribution; as a result, the liquid-vapor contact line is no longer sharp and clear because of capillary forces. The same phenomena can be also clearly observed in Figure 6(a)-6(b), where spatial variation of mass fraction of each species is displayed for both the case I and case II.

Our model can also predict saturation as a function of position. If all pores have one identical size (as in Case I), capillary pressure is the same in all pores, and as a result, we do not see the effect of capillarity and we thus expect that the saturation will be either 0 or 1 for both phases, which is shown in Figure 7(a). In the Case II with a spread distribution for the pore size, fluid saturation can take values between 0 and 1 due to the competition between capillary and gravity forces and the liquid-vapor contact region could be a transitional region instead of a sharp line. This is shown in Figure 7(b). With a sharp liquid-vapor contact in Case I, the overall mass density of the mixture (combining both phases) do have a jump across the liquid-vapor contact line, as demonstrated in Figure 8(a). On the other hand, with the mixing of phases in Case II, however, the overall mass density of the mixture varies 
continuously with depth, shown in Figure 8(b).

Since our model also computes pressure as a function of position. By comparing the generated saturation values and predicted pressure values at various positions, we can easily generate the curve of capillary pressure as a function of saturation (plotted in Figure 9). We note that the generated curve of capillary pressure as a function of saturation is highly sensitive to the assumed pore size distribution. We remark that as far as we know, all qualitative studies of capillary pressures in the literature are limited to fully immiscible two-phase/multi-phase fluids. It appears that our work in this paper is the first time in the literature to consider the capillarity of a two-phase fluid with partial miscibility and to predict the capillary pressure of partially immiscible fluids as a function of saturation.

\section{Concluding remarks}

In this paper, we establish a new energy-based framework to model multi-component twophase fluid systems at equilibrium at Darcy's scale. The focus of our model is the Helmholtz free energy. We adopt the Peng-Robinson equation of state (EOS) for the calculation of the bulk properties, and we model the gravity and capillarity effects by introducing terms into the total Helmholtz free energy expression. We show that the conventional compositional grading is a special case of our framework when one restricts it to spatial vertical dimension, together with the assumption of monodisperse pore-size distribution. However, our model works well with general distribution of pore size.

We derive and propose an unconditionally stable semi-implicit time marching scheme, which possesses the energy-decay property based on convex-concave splitting. We study two examples in the paper. The first example demonstrates the energy-decay property of our algorithm as applied to compositional grading of a binary fluid mixture consisting of carbon dioxide and normal decane. In the second example, we discuss more interesting physics about fluid mixture in a porous medium with general pore size distribution. In particular, we investigate the competition of capillarity and gravity, which also demonstrates the power of our model.

This model reported in this paper is the first work in the literature that rigorously incorporates capillarity and gravity effects into EOS-based phase equilibrium systems. In particular, it is the first time in the literature to model the capillarity of a two-phase fluid quantitatively with partial miscibility.

We observe that the curve of capillary pressure as a function of saturation as predicted by our model is highly sensitive to the assumed pore size distribution. In our numerical study here, only the log-normal distribution is considered for pore size distribution due to page limitations. An immediately extension of this work in near future is to use realistic pore size distributions.

Quite a lot of additional future work appears to be promising, which includes extension of current work to multiple spatial dimensions (2D and 3D), extension of current work to multi-component three-phase systems, and investigation of other EOS, especially the CPA (Cubic-Plus-Association) equation of state. This paper considers only conventional 
petroleum reservoirs; the extension of the model to unconventional reservoirs, tight reservoirs and shale will also be an interesting future work.

\section{Acknowledgements}

The work was supported in part by the research project given by King Abdullah University of Science and Technology (KAUST) through the grant BAS/1/1351-01-01.

\section{Appendix A. The two parameters in the Peng-Robinson equation of state}

In this appendix, we provide the details of the two parameters in the Peng-Robinson equation of state. The two parameters $a$ and $b$ can be computed as follows. For a mixture, these parameters can be calculated from the ones of the pure fluids by mixing rules:

$$
a(T)=\sum_{i=1}^{M} \sum_{j=1}^{M} y_{i} y_{j} a_{i}^{1 / 2} a_{j}^{1 / 2}\left(1-k_{i j}\right), \quad b=\sum_{i=1}^{M} y_{i} b_{i},
$$

where $y_{i}=n_{i} / n$ is the mole fraction of component $i$, and $a_{i}$ and $b_{i}$ are the Peng-Robinson parameters for pure-substance component $i$. For convenience, $k_{i j}$ is usually assumed to be constant for a fixed species pair.

Even though the pure-substance Peng-Robinson parameters $a_{i}$ and $b_{i}$ can be fit by using experimental data, they can also be computed from the critical properties of the species:

$$
\begin{aligned}
a_{i} & =a_{i}(T)=0.45724 \frac{R^{2} T_{c_{i}}^{2}}{p_{c_{i}}}\left(1+m_{i}\left(1-\sqrt{\frac{T}{T_{c_{i}}}}\right)\right)^{2}, \\
b_{i} & =0.07780 \frac{R T_{c_{i}}}{p_{c_{i}}} .
\end{aligned}
$$

As intrinsic properties of the species, the critical temperature $T_{c_{i}}$ and critical pressure $p_{c_{i}}$ of a pure substance are available for most substances encountered in engineering practice. In the above formula for $a_{i}$, we need also to specify the parameter $m_{i}$ for modeling the influence of temperature on $a_{i}$. It was suggested that one may correlates the parameter $m_{i}$ experimentally to the accentric parameter $\omega_{i}$ of the species by the following equations:

$$
\begin{aligned}
& m_{i}=0.37464+1.54226 \omega_{i}-0.26992 \omega_{i}^{2}, \quad \omega_{i}<0.5, \\
& m_{i}=0.379642+1.485030 \omega_{i}-0.164423 \omega_{i}^{2}+0.016666 \omega_{i}^{3}, \quad \omega_{i} \geq 0.5 .
\end{aligned}
$$

The accentric parameter can be fit by using experimental data, but if we lack data, we can also compute it by using critical temperature $T_{c_{i}}$, critical pressure $p_{c_{i}}$ and the normal boiling point $T_{b_{i}}$ :

$$
\begin{aligned}
\omega & =\frac{3}{7}\left(\frac{\log _{10}\left(\frac{p_{c_{i}}}{14.695 \mathrm{PSI}}\right)}{\frac{T_{c_{i}}}{T_{b_{i}}}-1}\right)-1 \\
& =\frac{3}{7}\left(\frac{\log _{10}\left(\frac{p_{c_{i}}}{1 \mathrm{~atm}}\right)}{\frac{T_{c_{i}}}{T_{b_{i}}}-1}\right)-1 .
\end{aligned}
$$




\section{Appendix B. Energy form of EOS vs. PVT form of EOS}

Equation (1) for the Helmholtz free energy $f_{b}(\mathbf{n})$ of a bulk fluid is known as an energyform of equation of state, and it has all the information stated in the PVT form of equation of state. In fact, it has more information than the corresponding PVT form, because the heat capacity information can also be derived from the Helmholtz free energy. Below we briefly show how one derives the PVT form of equation of state from the energy-form.

Based on the fundamental relation on thermodynamic variables, the pressure of homogeneous fluids $p$ and the Helmholtz free energy $f_{0}(\mathbf{n})$ can be linked in the following way

$$
\begin{aligned}
p & =p(\mathbf{n}, T)=-\left(\frac{\partial F_{b}(\mathbf{n}, T, V)}{\partial V}\right)_{T, \mathbf{N}} \\
& =-\left(\frac{\partial\left(f_{b}\left(\frac{\mathbf{N}}{V}, T\right) V\right)}{\partial V}\right)_{T, \mathbf{N}} \quad-f_{b}\left(\frac{\left.\partial \frac{N_{i}}{V}\right)_{N_{i}}}{\partial V}\left(\frac{\partial f_{b}}{\partial n_{i}}\right)_{T, n_{1}, \cdots, n_{i-1}, n_{i+1}, \cdots n_{M}} \sum_{i=1} f_{b}-V \sum_{i=1}^{M} n_{i}\left(\frac{\partial f_{b}}{\partial n_{i}}\right)_{T, n_{1}, \cdots, n_{i-1}, n_{i+1}, \cdots n_{M}}-\right. \\
& =\sum_{i=1}^{M} n_{i} \mu_{i}-f_{b} .
\end{aligned}
$$

We now substitute the Peng-Robinson expression of the Helmholtz free energy (1) into the above equation to conclude

$$
\begin{aligned}
p & =\frac{n R T}{1-b n}-\frac{n^{2} a(T)}{1+2 b n-b^{2} n^{2}} \\
& =\frac{R T}{v-b}-\frac{a(T)}{v(v+b)+b(v-b)} \\
& =\frac{R T}{v-b}-\frac{a(T)}{(v+b)^{2}-2 b^{2}} .
\end{aligned}
$$

The reason why people call it a "cubic" EOS is from the viewpoint of solving for the molar volume $v=1 / n$. For a given pressure, if we would like to solve for the molar volume $v=1 / n$, then the EOS can be re-arranged into an equation involving a cubic algebraic function of $v$.

\section{References}

[1] B. Breure and C.J. Peters. Modeling of the surface tension of pure components and mixtures using the density gradient theory combined with a theoretically derived influence parameter correlation. Fluid Phase Equilibria, 2012. 
[2] H.T. Davis. Statistical mechanics of phases, interfaces, and thin films. VCH, New York, 1996.

[3] C. Dawson, S. Sun, and M.F. Wheeler. Compatible algorithms for coupled flow and transport. Computer Methods in Applied Mechanics and Engineering, 193:2565-2580, 2004.

[4] X. Fan, J. Kou, Z. Qiao, and S. Sun. A component-wise convex splitting scheme for diffuse interface models with Van der Waals and Peng-Robinson equations of state. SIAM Journal on Scientific Computing, 39(1):B1B28, 2017.

[5] A. Firoozabadi. Thermodynamics of hydrocarbon reservoirs. McGraw-Hill, New York, 1999.

[6] D. Frenkel and B. Smit. Understanding molecular simulation: from algorithms to applications. Academic press, 2001.

[7] K.B. Haugen and A. Firoozabadi. Composition at the interface between multicomponent nonequilibrium fluid phases. J. Chem. Phys., 130:064707, 2009.

[8] J. Kou and S. Sun. Convergence of discontinuous galerkin methods for incompressible two-phase flow in heterogeneous media. SIAM Journal on Numerical Analysis, 51:3280-3306, 2013.

[9] J. Kou and S. Sun. Numerical methods for a multi-component two-phase interface model with geometric mean influence parameters. SIAM Journal on Scientific Computing, 37(4):B543-B569, 2015.

[10] J. Kou and S. Sun. Multi-scale diffuse interface modeling of multi-component two-phase flow with partial miscibility. Journal of Computational Physics, 318:349-372, 2016.

[11] J. Kou and S. Sun. Unconditionally stable methods for simulating multi-component two-phase interface models with peng-robinson equation of state and various boundary conditions. Journal of Computational and Applied Mathematics, 291(1):158-182, 2016.

[12] J. Kou and S. Sun. Efficient energy-stable dynamic modeling of compositional grading. International Journal of Numerical Analysis and Modeling, 14(2):218-242, 2017.

[13] J. Kou and S. Sun. A stable algorithm for calculating phase equilibria with capillarity at specified moles, volume and temperature using a dynamic model. Fluid Phase Equilibria, 456:7-24, 2018.

[14] J. Kou and S. Sun. Thermodynamically consistent modeling and simulation of multi-component twophase flow with partial miscibility. Computer Methods in Applied Mechanics and Engineering, 331:623649, 2018.

[15] J. Kou and S. Sun. Thermodynamically consistent simulation of nonisothermal diffuse-interface twophase flow with peng-robinson equation of state. Journal of Computational Physics, 371:581-605, 2018.

[16] J. Kou, S. Sun, and X. Wang. Efficient numerical methods for simulating surface tension of multicomponent mixtures with the gradient theory of fluid interfaces. Computer Methods in Applied Mechanics and Engineering, 292:92-106, 2015.

[17] J. Kou, S. Sun, and X. Wang. Linearly decoupled energy-stable numerical methods for multi-component two-phase compressible flow. SIAM Journal on Numerical Analysis, 56(6):3219-3248, 2018.

[18] L. W. Lake. Enhanced oil recovery. Prentice Hall, Englewood Cliffs, New Jersey, 1989.

[19] Y. Li, J. Kou, and S. Sun. Thermodynamically stable two-phase equilibrium calculation of hydrocarbon mixtures with capillary pressure. Industrial and Engineering Chemistry Research, 57(50):17276-17288, 2018.

[20] C. Miqueu, B. Mendiboure, A. Graciaa, and J. Lachaise. Modelling of the surface tension of pure components with the gradient theory of fluid interfaces: a simple and accurate expression for the influence parameters. Fluid Phase Equilibria, 207(1):225-246, 2003.

[21] C. Miqueu, B. Mendiboure, A. Graciaa, and J. Lachaise. Modeling of the surface tension of multicomponent mixtures with the gradient theory of fluid interfaces. Industrial \& engineering chemistry research, 44(9):3321-3329, 2005.

[22] H. Montazeri, S.H. Zandavi, and A. Bazylak. Sharp interface models for two-phase flows: Insights towards new approaches. Computer Methods in Applied Mechanics and Engineering, 322:238-261, 2017.

[23] J. Moortgat, S. Sun, and A. Firoozabadi. Compositional modeling of three-phase flow with gravity using higher-order finite element methods. Water Resources Research, 47:W05511, 2011.

[24] L.C. Nielsen, I.C. Bourg, and G. Sposito. Predicting CO2-water interfacial tension under pressure and 
temperature conditions of geologic CO2 storage. Geochimica et Cosmochimica Acta, 81:28-38, 2012.

[25] A. Onuki. Phase Transition Dynamics. Cambridge University Press, 2002.

[26] D.-Y. Peng and D.B. Robinson. A new two-constant equation of state. Industrial and Engineering Chemistry Fundamentals, 15(1):59-64, 1976.

[27] Z. Qiao and S. Sun. Two-phase fluid simulation using a diffuse interface model with Peng-Robinson equation of state. SIAM Journal on Scientific Computing, 36(4):B708-B728, 2014.

[28] L. Rongy, K.B. Haugen, and A. Firoozabadi. Mixing from fickian diffusion and natural convection in binary non-equilibrium fluid phases. AIChE J., 58(5):1336-1345, 2012.

[29] M. Struwe. Variational methods: applications to nonlinear partial differential equations and Hamiltonian systems, volume 34. Springer, 2008.

[30] S. Sun. Energy stable simulation of two-phase equilibria with capillarity. Rodrigues J. et al. (eds) Computational Science ICCS 2019. ICCS 2019. Lecture Notes in Computer Science, 11539, 2019.

[31] S. Sun and J. Geiser. Multiscale discontinuous galerkin and operator-splitting methods for modeling subsurface flow and transport. International Journal for Multiscale Computational Engineering, $6(1): 87-101,2008$.

[32] S. Sun and M.F. Wheeler. Symmetric and nonsymmetric discontinuous galerkin methods for reactive transport in porous media. SIAM Journal on Numerical Analysis, 43(1):195-219, 2005.

[33] S. Sun and M.F. Wheeler. Local problem-based a posteriori error estimators for discontinuous galerkin approximations of reactive transport. Computational Geosciences, 11(2):87-101, 2007.

[34] G. Tegze, T. Pusztai, G. Toth, L. Granasy, A. Svandal, T. Buanes, T. Kuznetsova, and B. Kvamme. Multiscale approach to CO2 hydrate formation in aqueous solution: phase field theory and molecular dynamics. nucleation and growth. J. Chem. Phys., 124:234710, 2006.

[35] J. D. van der Waals. "verhandel. konink. akad. weten. amsterdam" vol. 1 no. 8 (dutch) 1893, translation of j.d. van der waals (the thermodynamic theory of capillarity under the hypothesis of a continuous density variation). J. Stat. Phys., 1979.

[36] Y. Wang, S. Sun, L. Gong, and B. Yu. A globally mass-conservative method for dual-continuum gas reservoir simulation. Journal of Natural Gas Science and Engineering, 53:301-316, 2018.

[37] Y. Wang, S. Sun, and B. Yu. Acceleration of gas flow simulations in dual-continuum porous media based on the mass-conservation POD method. Energies, 10(9):1380, 2017.

[38] Y. Wang, Y. Wang, and Z. Cheng. Direct numerical simulation of gas-liquid drag-reducing cavity flow by the VOSET method. Polymers (Basel), 11(4):596/1-17, 2019.

[39] Y. Wang, B. Yu, and Y. Wang. Acceleration of gas reservoir simulation using proper orthogonal decomposition. Geofluids, Volume 2018(Article ID 8482352):1-15, 2018.

[40] H. Yang, S. Sun, Y. Li, and C. Yang. A scalable fully implicit framework for reservoir simulation on parallel computers. Computer Methods in Applied Mechanics and Engineering, 330:334-350, 2018.

[41] H. Yang, S. Sun, Y. Li, and C. Yang. A fully implicit constraint-preserving simulator for the black oil model of petroleum reservoirs. Journal of Computational Physics, 396:347-363, 2019.

[42] H. Yang, C. Yang, and S. Sun. Active-set reduced-space methods with nonlinear elimination for twophase flow problems in porous media. SIAM Journal on Scientific Computing, 38(4):B593-B618, 2016.

[43] Y. Yang, A.K.N. Nair, and S. Sun. Molecular dynamics simulation study of carbon dioxide, methane, and their mixture in the presence of brine. Journal of Physcial Chemistry B, 121(41):9688-9698, 2017. 